\title{
Die Rolle der Geographie in der Gesellschaft
}

\author{
Bericht und Referate zum Schweizerischen Geographentag vom 2./3. Mai in Bern
}

\section{Einleitung und Problemstellung (K. Aerni, Bern)}

\section{Die Rolle der Geographie in der Gesellschaft: Leidbild - Leitbild}

Hinter der nachgestellten Doppelbezeichnung Leidbild/Leitbild steckt das Eingeständnis, daß wir im Rahmen der Fachdisziplin Geographie eine Reihe von Problemen erkennen, die wir lösen möchten - in diesem Sinne gilt es, für die Schweizer Geographie ein Leitbild zu entwickeln. Folgende Feststellungen haben uns zu dieser Selbstbestimmung veranlaßt:

- Raum- und Umweltprobleme beschäftigen die Öffentlichkeit mehr denn je. Es ist daher wichtig, da $B$ die Geographie ihre Rolle und Aufgabe in diesem Zusammenhang vermehrt darstellt, bekanntmacht und wahrnimmt.

- Wer als Geograph/in beruflich tätig ist, spürt dauernd den Druck und die Kraft wohlorganisierter Berufsverbände. Es fehlt aber die "geographische Lobby».

- Auf wissenschaftspolitischer Ebene tritt die Geographie wenig effizient in Erscheinung. Gesuche von Geographen werden z. B. beim Nationalfonds ausschließlich von Nichtgeographen beurteilt.

- Die Kommunikation zwischen den Trägern der Geographie (Hochschule - Praxis - Schule) spielt nicht befriedigend.

Das Konzept zur Bearbeitung der sich stellenden Probleme wurde in einer Arbeitsgruppe «Strategie des Faches Geographie» entworfen, die unter der Leitung von Dr. Gilbert Thélin stand, dem Präsidenten der Schweizerischen Gesellschaft für Angewandte Geographie (SGAG). In ihr arbeiteten seit Sommer 1985 Geographen aus den Vorständen der Schweizerischen Geographischen Gesellschaft (SGgG), der Schweizerischen Geographischen Kommission (SGgK) und verschiedenen geographischen Instituten und Fachgesellschaften. In einer nächsten Phase wurden Arbeitsgruppen zu den Themen «Selbstbild und Fremdbild der Geographie und der Geographen», «Wissenschaft und Methoden»,
«Didaktik», "Wissenschaftspolitik» und «Umsetzung in Praxis und Politik» gebildet. Die folgenden Abschnitte bringen nun Referate aus verschiedenen Perspektiven, die aus eigenen Stellungnahmen, Gruppendiskussionen und ausgewerteten Umfragen entstanden sind. Abschließend folgt die Darlegung der einzuleitenden Maßnahmen im Sinne von Empfehlungen und Anträgen zuhanden der $\mathrm{SGgG}$, der SGgK, der Fachgesellschaften und der Hochschulinstitute.

Ein kurzer Rückblick zeigt, daß die Schweizer Geographie eine Selbstbestimmung auf breiter Basis nötig hat. Vor 30 Jahren existierten die einleitend formulierten Probleme noch kaum. Die Schweizer Geographie war damals sehr stabil und wurde vor allem von wenigen Lehrstuhlinhabern - die sich persönlich gegenseitig wenig kannten - und einer groBen Zahl von Schulgeographen repräsentiert. Das Berufsziel war einfach - die Ausbildung von Lehrern verschiedener Stufen war ja der Hauptauftrag der in den letzten 100 Jahren entstandenen geographischen Institute. Seither sind große Wandlungen eingetreten:

1. Die Geographie hat inzwischen im Rahmen der Planung an praktischer Bedeutung gewonnen, und die Hochschulinstitute sind bezüglich Lehrpersonal und Infrastruktur gewachsen.

2. Der traditionelle Absatzmarkt für unsere Absolventen, die Lehrtätigkeit in Schulen verschiedener Stufen, ist ais Folge der demographischen Veränderungen massiv geschrumpft, vor allem auf der Stufe der höheren Mittelschulen.

3. Unsere Absolventen müssen ihr Auskommen als angewandte Geographen, als Geographen in der Nichtschul-Praxis finden. Der Berufs-Geograph ist demnach das Ausbildungsziel, auf das sich die Universität besinnen muß.

Folgerung:

Früher basierte das wissenschaftliche Wachstum der Hochschulgeographie in weiten Teilen auf der Karriere der Schulgeographen. Heute entsteht entscheidendes neues geographisches Wissen und Können in der Berufspraxis, außerhalb der Studierzimmer von Mittelschul- und Hochschullehrern. 


\section{Einführungsreferate}

2.1 Roswitha Hantschel, Prof., Dr., Universität Frankfurt a. M.: Neue Wege der Geographie in Deutschland

2.2 Claude Raffestin, Prof., Dr., Université de Genève: De l'idéologie à l'utopie ou la pratique du géographe

2.3 Charles Hussy, Dr., Université de Genève: Aspects internes et externes d'une identité

\subsection{Schweizer Geographie am Wendepunkt}

A) Klaus Aerni, Prof., Dr., Universität Bern,

B) BennoWerlen, Dr., Universität Zürich-Irchel,

C) Martin Boesch, Dr., Dozent Handelshochschule St. Gallen:

\subsection{Roswitha Hantschel: Neue Wege der Geographie in Deutschland}

Sie haben mir die Aufgabe gestellt, neue Wege der Geographie in Deutschland aufzuzeigen - und dies in einem Tagungsrahmen, der sich mit der Rolle der Geographie in der Gesellschaft beschäftigen soll. Beides scheint mir aus einer Art "Aufbruchstimmung» zu resultieren, die sich nicht damit zufrieden geben will, der Kritik am Fach noch eine weitere hinzuzufügen, sondern einen methodischen Rahmen und Arbeitsstrategien fürWeiterentwicklungen erarbeiten möchte. Natürlich entsteht ein solcher Wunsch aus einem Unbehagen, aus Kritik, die innerhalb der Geographie entstanden ist und die von auBen an sie herangetragen wird. Aber jede Unzufriedenheit trägt gleichzeitig Lösungsansätze in sich, denn sie mißt das Vorgefundene an dem, was sein könnte und sollte. In diesem Sinne möchte ich im folgenden versuchen, die Kritik auf ihren Gegenpol zu bringen und Wege oder Ansatzpunkte für Veränderungen zumindest anzudeuten. Um die verschiedenen Positionen kritischer Äußerungen deutlich zu machen, seien an den Anfang drei Zitate gestellt: BUTZIN (1982, 94) unterscheidet zunächst drei Schwerpunkte innerhalb des Faches selbst. Es geht dabei um:

1. disziplinpolitische «Überlebensstrategien» (er bezeichnet dies als «besondere Erscheinung der deutschen Geographie»);

2. die «Methodischen Auseinandersetzungen auf der (vermeintlich?) gemeinsamen Basis des Kri- tischen Rationalismus (z. B. die WIRTH-DÜRRAuseinandersetzung: DÜRR 1979, WIRTH 1980)» und

3. «wird die forschungslogische (szientistische) Basis selbst kritisiert und ersetzt wie in den phänomenologischen und marxistischen Ansätzen (u. a. PEET 1977, HARVEY 1977) oder es werden einzelne, in ihr entwickelte Paradigmen, wie der „spatial” und „behavioral approach" in Frage gestellt ...

Gemeinsam scheint diesen Positionen das Unbehagen darüber zu sein, daß die gebräuchlichen Lösungsmuster und wissenschaftlichen Fragestellungen angesichts bestimmter realweltlicher Probleme versagen (vgl. PEET 1977, GREGORY 1978, BLUNDEN et al. 1978, SCHMIDT-WULFFEN 1980) bzw. sie sogar erhalten und verstärken (OLSSON 1977).» Dieser mangelnde Praxisbezug wird auch in der Broschüre "Informationen in eigener Sache» der Schweizerischen Gesellschaft für Angewandte Geographie 1985 angesprochen: "Im Hinblick auf die anhaltende Zerstörung und Veränderung von Landschaft, Kultur- und Lebensraum sowie angesichts anstehender und ungelöster Umwelt-, Regional- und Entwicklungsprobleme erwarten Geographen von der Geographie, daß sie sich in nächster Zukunft vermehrt in Problemlösungsprozesse einschaltet.» Hier möchte ich allerdings hinzufügen, da $\beta$ es hoffentlich nicht nur die Geographen erwarten, sondern auch die Öffentlichkeit, die Gesellschaft, denn sonst wäre das Fach tatsächlich überflüssig. Aber dem positiven Anliegen entsprechend kann man nun auch wieder umgekehrt argumentieren: Wenn man der allgemeiner formulierten Aussage zustimmt, daß die Gesellschaft von der Wissenschaft Lösungsansätze erwartet, dann ergeben sich zwei Aufgaben von existenzieller Bedeutung für die Geographie: Sie muß erstens zeigen, da $ß$ sie zu den Wissenschaften zählt, die von den angesprochenen Fragestellungen betroffen sind, und zweitens muß sie nicht nur Strukturen beschreiben, sondern auch Probleme in ihrer Entstehung erklären und damit Ansatzpunkte für Lösungsstrategien bieten. Auf die methodologischen Konsequenzen dieser Forderung komme ich noch zurück. Zunächst möchte ich noch ein drittes Zitat anfügen, das in wenigen Worten eine ganze Reihe fachinterner Diskussionen zum Forschungsgegenstand, zur Spezialisierung der Disziplinen und zum vermeintlichen Gegensatz zwischen Physischer Geographie und Humangeographie zusammenfaßt:

LEUTZINGER schreibt in der Geographica Helvetica (1984, Nr. 2, S. 107): "Geographen leiden. Sie leiden an ihrem Gegenstand, der sich nicht verbindlich definieren läßt und obendrein nicht 
ihnen allein gehört. Geographen leiden an der Aufteilung der Wissenschaft, denn ihr Fach ... ist keine exakte Naturwissenschaft - und bei den Geisteswissenschaften nimmt man sie nicht ernst.»

Gerade hier sehe ich allerdings eine Chance für die Geographie, nun nicht wieder - wie so oft - wissenschaftstheoretische Tendenzen in anderen Disziplinen abzuwarten und sie mit erheblicherVerspätung, sozusagen «aus zweiter Hand», zu übernehmen ebenso wie Verfahren und Techniken, die dann für das Fach «zurechtgebogen» werden. Es sollte vielmehr mit durchaus angemessenem Selbstbewußtsein deutlich gemacht werden, daß der Weg zur Eigenständigkeit der Geographie nicht darin besteht, zur «reinen» Natur- oder Geisteswissenschaft (bzw. Gesellschaftswissenschaft) zu werden und zur Erreichung dieses Ziels eventuell endgültig in zwei entsprechende Teile zu verfallen, sondern darin, da $B$ sie beide Aspekte betrachtet -, und zwar nicht nur nebeneinander, sondern in ihrem Zusammenwirken. Der Zeitpunkt für eine solche Neubesinnung ist sicher günstig, denn Unbehagen wird in und an vielen Wissenschaftsbereichen geäußert; wir stehen - hoffentlich - am Beginn einer "Wendezeit», wie CAPRA es nennt: die Methoden der sogenannten «exakten» Naturwissenschaften erweisen sich nämlich zunehmend als nicht ausreichend für die Lösung vieler aktueller Probleme, und auch die Gesellschaftswissenschaften sind mehr und mehr der Kritik ausgesetzt, vor allem dort, wo die Umsetzung ihrer ökonomischen Modelle eher Probleme verursacht als sie zu lösen oder wo einseitig gesellschaftsbezogene Vorstellungen ökologische Schranken mißachten. Nun stellt sich allerdings die Frage, ob ein Fach überhaupt in der Lage ist, eine solche Integration zu leisten, und dabei weder in «idiographische» noch «szientistische» Fehler zurückzufallen. Gerade die Diskussion um die Wissenschaftlichkeit "ganzheitlicher» Ansätze (etwa der Länderkunde) hatte ja zunächst in nur eine Richtung geführt: die des Kritischen Rationalismus, der sich an der Methodologie der Naturwissenschaften orientiert. Ob die Ursache darin liegt, daß die ersten deutschen Geographen, die diese Diskussion vor etwa 20 Jahren überhaupt aufgriffen (z. B. BARTELS 1968), hier ein Schwergewicht setzten oder darin, daß durch die Physische Geographie dafür Potentiale innerhalb des Faches selbst vorhanden waren, mag dahingestellt bleiben. Jedenfalls waren durch diese Tendenz zu analytischen Methoden und die gleichzeitig ablaufende und damit in Begründungszusammenhang gebrachte - «Quantitative Revolution» die Voraussetzungen für immer stärkere Spezialisierung und damit auch Auseinanderdriften von Physischer und Humangeographie gegeben.

SEDLACEK bemerkt noch 1982 (S. 13) zum Verhältnis beider Teile: «Daneben ist es beim heutigen Stand der Wissenschaften subjektiv unmöglich, in beiden Bereichen angemessene Leistungen zu erbringen. Selbst in jenen Jahren, als das szientistische Postulat einer wissenschaftlichen Einheitsmethode weithin anerkannt wurde und systemtheoretisches Denken gewichtig war, lief die Entwicklung von Kulturund Naturgeographie weiter auseinander. Solange die mythisch gepflegte Klammer einer „Raumwissenschaft" wissenschaftstheoretisch nicht begründet werden kann, und diese Möglichkeit ist sowohl nach dem Scheitern des ,spatial approach” als auch nach dem jüngsten fehlgeschlagenen Versuch einer wissenschaftstheoretischen Begründung der Länderkunde durch wIRTH (1978b) nicht in Sicht, muß der Dualismus beider Fachrichtungen als unüberwindbar gelten.»

Ich kann mich dieser Meinung nicht anschließen. Im Gegenteil: ich sehe in der noch bestehenden Verbindung zwischen Physischer und Humangeographie - wenn sie auch im Augenblick oft mehr institutionell als inhaltlich ist - eine Chance für die Weiterentwicklung des Faches, und zwar unter drei Aspekten:

- Veränderung des Anwendungsbezugs zum Handlungsbezug

- Erweiterung des systemanalytischen Ansatzes durch den der Systemsteuerung

\section{- Einbezug normativer Theorien in die Forschung.}

Diese Punkte zeigen schon, da $B$ ich mein Vortragsthema nicht als eine Bestandsanalyse augenblicklicher Ansätze in der deutschen Geographie auffasse. Dazu gibt es bereits Arbeiten, und ich möchte nochmals einige Passagen aus dem Sammelband «Kultur-/Sozialgeographie» zitieren, in dem SEDLACEK als Herausgeber versucht, ein Bild des Diskussionsstandes zu vermitteln:

Neben der Kritik am Szientismus und am Verhaltenskonzept «entwickelten sich sozialkritische Ansätze einer neuen ,humanistic geography” aus dialektischen und philosophischen Schulen (vgl. etwa PEET 1977, GREGORY 1978, GALE/OLSSON (eds.) 1979, SCHMIDT-WULFFEN 1980). Mittlerweile hat diese Bewegung auch auf die Bundesrepublik Deutschland übergegriffen und nicht nur zu einer Rehabilitation der Anfang der siebziger Jahre szientistisch verdrängten Positionen, sondern auch zu ersten Ansätzen eines neuen fachtheoretischen Diskurses in der Geographie geführt. Nimmt man solche Auffassungen hinzu, die beim szientistischen Umbruch der Landschafts- und Länderkunde in der fachtheoretischen Diskussion untergetaucht sind und" heute in Form einer «wissenschaftstheoretischen Begründung der Länderkunde» (WIRTH 1978) oder einer "Ökogeographie» (LESER 1980) «erneut zutage treten, ... dann läßt sich für die Gegenwart ein erheblicher Pluralismus in der geographischen Fachtheorie feststellen» (S. 11f.). 
Statt alle diese Positionen nebeneinander zu referieren, möchte ich im folgenden von vornherein Schwerpunkte setzen, und zwar dort, wo der eigene wissenschaftstheoretische Standort einen Begründungszusammenhang liefern kann und wo sie - meiner Meinung nach - Zukunftsperspektiven für eine enge Verbindung von Geographie und Praxis bieten. Die Betonung der eigenen Subjektivität in diesem Zusammenhang soll zum einen darauf hinweisen, daß dieser Vortrag bei einem anderen Vertreter der deutschen Geographie - etwa mit kritisch-rationalistischer Wissenschaftsauffassung - natürlich ganz anders aussehen würde, zum anderen aber auch darauf, daß wissenschaftstheoretische Überlegungen und solche zum Verhältnis von Theorie und Praxis ganz wesentlichen Einfluß auf die weitere Entwicklung des Faches haben werden.

Dies drückt sich auch in dem ersten genannten Aspekt aus: Handlungsbezug der Geographie statt Anwendungsbezug. In diesen beiden Begriffen stekken grundlegend unterschiedliche Auffassungen über den wissenschaftlichen Anspruch des Faches. Wenn man nämlich Wissenschaft als Hilfe zur Daseinsbewältigung und zur Verbesserung der Lebensumstände versteht, dann reicht die Erarbeitung von Aussagen über "Regelhaftigkeiten" nach dem Vorbild von Naturgesetzen in der Geographie allein nicht aus, und man kann die «Umsetzung» solcher beschreibenden Erkenntnisse auch nicht aus dem Forschungsbereich herausnehmen und an Politik und Planung verweisen. Dies wird besonders deutlich, wenn man etwa räumliche Erscheinungen betrachtet, in denen sich vorwiegend menschliche Aktivitäten ausdrücken. Nehmen wir als Beispiel die Wohnstandorte in einem städtischen Gebiet. Hier wurde mit verschiedenen Ansätzen versucht, zeitliche und räumliche Abläufe als «regelhaft» zu erfassen: indem die soziale Segregation in Wohngebieten unterschiedlicher Qualität der Steuerung durch Gruppen bzw. durch deren Merkmale zugeschrieben wurde (Münchner Sozialgeographie), dem Einflu $ß$ der individuellen oder ebenfalls gruppengebundenen Wahrnehmung und Bewertung des Wohnungsangebots (Perzeptionsansatz), den Auswirkungen von "constraints» in zeitlicher, technischer oder ökonomischer Hinsicht (Aktionsraumforschung) usw. Mit dem Erkennen solcher empirischen Zusammenhänge wurde oft gleichzeitig der Anwendungsbezug der Geographie zu belegen versucht. Sie sollte "Grundlagenmaterial» für die Planung liefern $-z$. B. in bezug auf Infrastrukturausstattung eines Wohngebiets der gehobenen Mittelschicht, die vorhanden sein muß, wenn etwa eine Stadt diese steuerkräftige Bevölkerung nicht durch Abwanderung verlieren will. Aber kann man dabei von Daseinsbewältigung oder gar Verbesserung der Lebensumstände sprechen? Was geschieht $\mathrm{z}$. B. mit der Bevölkerung, die im sogenannten «Wohnungsmarkt» die schwächste Position hat? Nimmt sie wirklich die schlechter ausgestatteten Standorte ein, weil sie Wohnqualität geringer bewertet? Bei dieser Art der Betrachtung steht im Hintergrund vielleicht der aus älteren Weltbildern übernommene Harmonisierungsgedanke, nach dem sich im Raum ein «Gleichgewicht» der Kräfte und Funktionen einpendelt, entsprechend dem natürlichen Auswahlprinzip der Evolutionstheorie. Abgesehen davon, $\mathrm{da} ß$ auch dem Gleichgewichtsbild im anorganischen und biologischen Bereich eine gewisse romantische Verklärung anhaftet, bedarf es zumindest bei seiner Übertragung auf menschliche Gesellschaften einer kritischen Reflexion. Denn das Auswahlprinzip, z. B. bei der Konkurrenz um bestimmte Standorte, unterliegt ja keinen Naturgesetzen, sondern Kriterien, die sich Menschen während ihrer geschichtlichen Entwicklung selbst gesetzt haben - etwa dem Primat ökonomischer Kalkulation oder wirtschaftlichen Wachstums.

Damit sind solche Prinzipien aber gleichzeitig grundsätzlich veränderbar; aus der naturwissenschaftlichen Frage «warum?», deren Antworten sich nur technologisch umsetzen, d. h. anwenden lassen, wird die handlungsbezogene Frage "warum eigentlich?», die zu Veränderungen der vermeintlich erklärenden Variablen selbst führen kann.

Wenn heute zunehmend erkannt wird, daß die sich durchsetzenden Kräfte und Interessen weder «natürlich» noch unbedingt - in größeren Zusammenhängen betrachtet - «wünschenswert» sind, dann heißt das nicht, daß Konflikte in der Raumnutzung erst jetzt auftreten. Es gab sie wohl in der gesamten menschlichen Geschichte (und im Grunde auch während der «biologischen» Evolution); aber da auch die Wissenschaft in die jeweiligen zeitlichen Welt- und Menschenbilder eingebunden ist, blieb zumindest der Aspekt der Verantwortung der Forschung für die Lösung solcher Konflikte wenig beachtet. Doch angesichts der nun wohl erstmals so deutlich zutage tretenden Möglichkeit einer existenziellen Bedrohung des Menschen - und zwar durch sein eigenes Einwirken - sollt die Notwendigkeit eines Handlungsbezugs gerade in die Methodologie der Geographie einsichtig sein.

Natürlich brauchen wir auch technologisches Wissen zur Daseinsbewältigung, und viele Bereiche der Geographie - vor allem der Physischen Geographie - können es erarbeiten. Aber bereits bei Zusammenhängen, die wir zwar erkennen und uns danach richten, aber nicht verändern können, also bei wirklichen Naturgesetzen, ist zu reflektieren, wie wir damit umgehen und welche Auswirkungen ihre Anwendung hat. Überall dort, wo menschliche Einflüsse wirken, ist also grundsätzlich die Frage nach der Zwangsläufigkeit der untersuchten Erscheinungen zu stellen - und hier sind weite Teile der Physischen Geographie ebenso angesprochen wie die $\mathrm{Hu}$ mangeographie. 
Der Raum erscheint somit in einer neuen Dimension, nämlich als Spannungsfeld von Nutzungsinteressen und Konflikten, wobei wir weder davon ausgehen müssen, daß seine naturräumliche Ausstattung die Nutzung dominiert, noch davon ausgehen können, daß das «technisch Machbare» uneingeschränkt umsetzbar ist.

Zwar erscheint uns die Trennung von Mensch einerseits und Umwelt andererseits sehr selbstverständlich, weil sie sich in langer wissenschaftlicher Tradition geformt hat und sich nicht nur in der Dichotomie von Natur- und Gesellschaftswissenschaften ausdrückt, sondern auch in der von Physischer und Humangeographie und allen ihren bisherigen Konzepten zur Beschreibung und Erklärung von Raumstrukturen. Aber wenn wir diese Trennung einmal gedanklich aufgeben und die Untersuchungseinheiten anders abgrenzen - nämlich als Mensch-RaumHandlungseinheiten, die sich teilweise ergänzen und in Austausch treten, größtenteils aber miteinander um Nutzung und Verfügung konkurrieren dann stellt sich der Geographie eine neue Aufgabe. Sie muß nicht nur erkennen, da $\beta$ auf den verschiedenen gesellschaftlichen Ebenen (z. B. Individualebene - Organisationsebene - Gesellschaftsebene; kommunal - national - international; Minoritäten Majoritäten usw.), aber auch etwa zwischen wirtschaftlichen und gesundheitlichen Interessen, neben unterschiedlichen Ansprüchen auch unterschiedliche Verfügungs- und Handlungsmöglichkeiten bestehen, die zu einem Auseinanderklaffen von «Bedürfnissen» und «Durchsetzung» führen.

Sie ist auch vor allem dann gefordert, wenn es darum geht, konkurrierende Interessen überhaupt erst offenzulegen $-z$. B. die Belange des Wasserhaushalts in einem städtischen Verdichtungsgebiet, die ansonsten nach dem Leitbild der «Verfügung über die Natur» meistens erst dann beachtet werden, wenn bereits Schäden auftreten, und zwar solche, die sich negativ auf die jeweils geltenden Leitprinzipien auswirken; oder die Ansprüche sozial Schwacher, die im «ökonomischen Marktmechanismus» die geringste Lobby haben und durchaus nicht freiwillig «höherwertigen» Raumnutzungen Platz machen. Die Beispiele ließen sich beliebig fortsetzen, wobei fast immer deutlich wird, daß sich solche Ansprüche eben nicht dominant in räumlichen Strukturen niederschlagen, sondern höchstens in Verdrängungsprozessen; d. h. die Untersuchung von Raummustern gibt zunächst nur Aufschluß darüber, welche Interessen sich - unter gegebenen gesellschaftlichen Bedingungen und Auffassungen über die Einheit bzw. Trennung Mensch-Umwelt durchgesetzt haben.

Wenn wir daran etwas ändern wollen, reicht erstens die Untersuchung solcher Raumstrukturen allein nicht aus, und zweitens müssen Leitbilder gefunden werden, an denen sich Veränderungen orientieren können.
Dies weist schon auf den dritten angesprochenen Aspekt für zukünftiges geographisches Arbeiten hin, aber zunächst möchte ich noch einen Gedanken fortführen, der sich aus der Forderung nach integrativer Betrachtung von konkurrierenden Mensch-Umwelt- bzw. Gesellschaft-Umwelt-Einheiten einerseits und dem nach Handlungsbezug andererseits ableiten läßt: die Erweiterung des systemanalytischen Ansatzes durch den der Systemsteuerung. Das Aufgreifen des Systemgedankens war naheliegend in einem Fach, das sich traditionell mit den Wechselwirkungen vieler Faktoren auf der Erde bzw. in Erdausschnitten auseinandersetzt. Es geschah jedoch zu einem Zeitpunkt, als analytische Methoden prägend waren, die Entwicklung von Physischer und Humangeographie auseinanderging und die Vorstellung der technischen Anwendung von Faktenwissen, der Beherrschbarkeit von Natur dominierte. Entsprechend präsentierten sich auch Versuche, Zusammenhänge in Fließdiagrammen darzustellen. Oft ist ein "natürlicher, primärer» und ein «gesellschaftlicher, sekundärer» Bereich getrennt, der Raum mit seiner Kultur- und Naturlandschaft erscheint als «Herausforderung», der sich die Gesellschaft stellt. Aber auch dort, wo beide Bereiche zusammengebracht werden, erscheint das «sekundäre Milieu» unter dem Aspekt der «sozioökonomischen Gesetzlichkeiten» und nur in einigen manifest gewordenen Abschnitten.

Die Vorteile des Systemansatzes, der wie alle wissenschaftlichen Methoden ein Versuch ist, Realität zu begreifen, werden dort deutlich, wo uns dieses Begreifen schwerfällt. So bemerkt KLAUS (1985, S. 6): «Unsere Gehirnleistungen wurden ganz offensichtlich nicht selektiert, um das Verhalten komplexer, nichtlinearer Systeme, so häufig diese auch in der Natur zu beobachten sind, zu prognostizieren. Vielmehr erwarten wir, ganz im Sinne einfacher linearer Systeme erster Ordnung, als Ergebnis eines kurzen Impulses einen verzögerten, vielleicht auch abgeschwächten oder verstärkten Impulsoutput. Komplexe Rückkopplungsketten vermuten die angeborenen Lehrmeister unseres Denkens nicht, da Menschen prinzipiell von der Hypothese der Existenz einfacher Lösungen ausgehen.

... Gerade in dieser Tatsache . . liegt eine der entscheidenden Ursachen für die fortlaufende Veränderung der Umwelt durch den Menschen, ohne daß die Konsequenzen dieser Änderungen auch nur näherungsweise abgeschätzt werden.»

Die Schwierigkeiten liegen dort, wo mit dem analytischen Ansatz auch die Gleichsetzung von Beschreibung und Erklärung in den gesellschaftlichen Bereich hineingetragen wird. Ich möchte dies am Begriff des Systemzwecks erläutern. Wenn wir zunächst das "Ökosystem" - ohne die Existenz und das Einwirken des Menschen - betrachten, so zeigt sich, da $B$ das Zusammenwirken der Elemente von Atmosphäre, Hydrosphäre, Lithosphäre und Bio- 
sphäre immer wieder zu einem «dynamischen Gleichgewicht» (ROSNAY 1977, S. 26) führt. Die Begrenztheit der Gesamtmenge an «Material» und die Begrenztheit der Variationsmöglichkeiten der Eigenschaften anorganischer und organischer Komponenten setzt den Rahmen, in dem sich Veränderungen abspielen können, d.h. es stellt sich ein Gleichgewicht ohne bewußtes Zutun der beteiligten Elemente ein. Dieses Gleichgewicht, dessen Erhaltung als Systemzweck zu definieren ist, kann nicht mit menschlichen Maßstäben bewertet werden; es ist weder «gut» noch «schlecht», ebensowenig wie die Prozesse, die dabei ablaufen (etwa die Nahrungskette, die für Einzelindividuen ja Vernichtung bedeutet).

Kann man nun diese Definition des Systemzwecks auf Systeme übertragen, in denen Menschen bewu $\beta t$ handeln, in denen Ziele gesetzt werden, die von Bewertungen und Normen beeinflußt sind? Auf die damit verbundenen Probleme wurde schon hingewiesen: Die Bodennutzung in einem Stadtteil wird z. B. als das Ergebnis des Einwirkens ökonomischer Regelungsmechanismen betrachtet. Die Bodenpreise «selektieren» die Nutzer nach ihrer Zahlungsfähigkeit, es stellt sich ein dynamisches "Gleichgewicht» über Angebot und Nachfrage ein. Die Herstellung dieses Gleichgewichts ist aber nun durchaus nicht unbewußt, denn das Handeln nach ökonomischen Prinzipien bedeutet eine Orientierung an Normen. Das Verdrängen finanziell Schwächerer, das die Folge solcher Mechanismen ist, kann nicht als «natürlich» angesehen werden und widerspricht $\mathrm{z}$. B. sozialen Prinzipien. Im Zusammenhang mit menschlichen Handlungen kann also zumindest nicht von einem völlig wertneutral formulierten Systemzweck «Erhaltung des Gleichgewichts» ausgegangen werden, da die Prinzipien dieses Gleichgewichts (z. B. ökonomisch, sozial) von den Beteiligten selbst reflektiert, bewertet und beeinflußt werden.

Gesellschaft-Raum-System enthalten außer nichtorganischen und organischen Strukturen auch geistige Konstrukte, Sinngehalte. Wenn der Mensch als Element in Systemen betrachtet wird, ist also zu berücksichtigen, daß seine Handlungen nicht nur dem Ergebnis seiner physiologischen Evolution entsprechen, sondern auch den Einflüssen der gesamten gesellschaftlichen Entwicklung unterliegen, die Systemzwecke in Wechselwirkung mit vorgefundenen Raumstrukturen bewußt setzt.

Damit wird wiederum die Verantwortung des Menschen für sein (reflektiertes bzw. reflektierbares) Handeln deutlich: «Natürliche» und «gesellschaftliche» Systemelemente lassen sich zwar in bezug auf unbewußtes Reagieren und bewußtes Agieren differenzieren, aber sie stellen keine unabhängigen Einheiten dar, sondern sind in einem Gesamtsystem miteinander verflochten (wobei der Mensch selbst mit seinen physikalisch-chemisch-biologischen und seinen geistig-gesellschaftlichen Komponenten beiden Bereichen angehört). Diese Einbindungen müssen bei bewußt herbeigeführten Eingriffen berücksichtigt werden. Es ist weder angemessen, allein nach gesellschaftlich entwickelten Normen zu handeln und dabei Grenzen zu mißachten, die durch natürliche Zusammenhänge gesetzt sind (z. B. ökonomische «Ausbeutung» von Waldbeständen ohne Berücksichtigung klimatischer Folgen), noch ist es angemessen, gesellschaftliche Zusammenhänge wie «Mechanismen» zu betrachten und damit z. B. sozial negative Folgen (etwa die Benachteiligung Schwächerer) als «natürlich» zu definieren. Diese veränderte Betrachtung weist darauf hin, daß der Mensch ein Teil des Gesamtsystems ist, aus dem er sich nicht durch Willensakt lösen kann, dem gegenüber er aber durch seine Fähigkeit zur Reflektion eine Verantwortung trägt, eine Verpflichtung zur Steuerung (verändert nach: HANTSCHEL 1985, S. 14f.).

Dabei reichen analytische und quantifizierende Methoden allein nicht aus; die in szientistischem Eifer zeitweilig verdrängten ganzheitlichen Ansätze und qualitativen Aspekte erlangen neue Bedeutung. Es kommt aber noch eine andere Aufgabe hinzu, die bereits mehrfach anklang: die Notwendigkeit des Einbezugs normativer Überlegungen in die Forschung selbst, und zwar an zwei Stellen. Erstens gibt nur die Kenntnis der Faktoren, ihres Zusammenwirkens in Prozessen und der dahinter stehenden menschlichen Wertungen Hinweise auf Steuerungsinstrumente, die entweder zur Erhaltung oder zur Veränderung der analysierten Verhältnisse eingesetzt werden können. Zweitens können für die Entscheidungsfindung zur Erhaltung oder Veränderung - wenn man diese überhaupt aus dem Forschungsbereich ausklammern will - nur dann Alternativen aufgezeigt werden, wenn Werte und Normen offengelegt werden, die entweder koexistieren (z. B. in unterschiedlichen Gesellschaft-Raum-Systemen), die miteinander konkurrieren (z. B. in Raumnutzungskonflikten) oder die zugunsten dominanter Muster verdrängt werden (z. B. ökonomisches vor ökologischem Prinzip).

Bisher hat man solche allgemeinen Wertüberlegungen, analog zur übrigen Aufspaltung der Disziplinen, einem streng abgegrenzten Teilbereich - der Philosophie - überlassen, der das Ganze des Daseins, Sinnbezüge, Grundwerte u. ä. betrachtet, ohne aber gezielt auf konkrete zeitlich und räumlich gebundene Phänomene einzugehen. Er steht damit sowohl institutionell als auch inhaltlich neben den anderen Wissenschaftsbereichen - teilweise belächelt und in seiner Wissenschaftlichkeit in Frage gestellt, höchstens geduldet, aber durchweg ohne realen Handlungsbezug. Genau dies würde sich aber ändern, wenn Werte und Normen ein Prüfkriterium für empirische Forschung darstellen. 
Natürlich stellt sich hier die Frage, ob philosophische Ansätze selbst zum Gegenstand geographischer Forschung werden können und sollen. Ich selbst möchte diese Frage bejahen, und zwar in der Form, daß sie um den räumlichen Aspekt erweitert werden. Die meisten sozial- bzw. moralphilosophischen Überlegungen der Vergangenheit und Gegenwart reflektieren zwar unterschiedliche Ausgangsbedingungen für die Entwicklung eines Menschen im individuellen Bereich - also etwa die verschiedene Ausstattung mit geistigen und körperlichen Kräften, die soziale Herkunft, ökonomische Startbedingungen usw. Es fehlen aber Unterschiede, die über das Individuelle hinausgehen und die sowohl im Bereich gesellschaftlicher Strukturen und Ebenen als auch in disparitären Eigenschaften des Raumes liegen bzw. durch die Wechselwirkung zwischen beiden entstanden sind und noch entstehen $-z$. B . physische Gegebenheiten, Endlichkeit von Ressourcen, Institutionen und Leistungen, überkommene Raumstrukturen und Standortpräferenzen usw. Auf dieser Grundlage kann diskutiert werden, wieweit und in welcher Form solche Unterschiede aufgehoben, kompensiert oder auch akzeptiert werden können, d. h. ich halte es für sinnvoll, etwa neben dem Begriff der sozialen Gerechtigkeit auch den der «räumlichen Gerechtigkeit» zu definieren (vgl. HANTSCHEL 1982).

Auch zu dieser Frage stellt SEDLACEK in seiner schon genannten Aufsatzsammlung unterschiedliche Positionen zusammen, und ich möchte wiederum eine Auswahl zitieren. So beziehen einige kritische Ansätze, z. B. des "welfare approach», Werturteile in ihre Aussagen ein, ohne sich aber bisher um eine methodische Ableitung von Normen bzw. Normenpräferenzen zu bemühen. SEDLACEK (1982, S. 188) fährt fort: "So stellt etwa SCHMIDT-WULFFEN (1980) . . . ausdrücklich die Notwendigkeit eines „Vorverständnisses" fest, dessen Rechtfertigung offen bleibt. Für die konstruktive Wissenschaftstheorie und ihre Anwendung auf materiale theoretische Leistungen der Fachwissenschaften ist dagegen charakteristisch, daß sie ... die Bildung des normativ-kritischen Wissens als vorrangiges Ziel handlungsorientierter Fachwissenschaften begreift.» Er bezieht sich damit auf LORENZEN (1978, S. 40), der sagt: «Normen und die Frage nach ihrer Gerechtigkeit (sind) das Hauptthema der konstruktiven Wissenschaftstheorie.» Da SEDLACEK wie erwähnt von einer notwendigen Trennung von Physischer und Humangeographie ausgeht, bezieht er diese Äußerung dann zunächst auch nur auf einen Teilbereich und formuliert (a. a. O. S. 197): «Wir können demgemäß von praktischen Aufgaben der Kulturgeographie sprechen, wenn es um die Begründung (Rechtfertigung) der zu verfolgenden Zwecke geht. Dies hat in Situationen zu erfolgen, in denen miteinander unverträgliche Zwecke des Handelns vorge- schlagen werden, deren gemeinsame Realisierung auch bei kluger Wahl der technischen Mittel ausgeschlossen bleibt.» Entsprechend dem dargestellten Anliegen, in der Geographie die Systembetrachtung und die Bildung von Gesellschaft-Umwelt-Einheiten weiterzuentwickeln, wäre diese Auffassung auf das gesamte Fach zu übertragen.

Zum Schluß noch einige Bemerkungen zur Öffentlichkeitsarbeit, die ja auch Gegenstand dieser Tagung sein soll. Ich meine, daß diese Reflektion der Öffentlichkeitsarbeit nicht nur der Selbstdarstellung dient, sondern auch aus der angesprochenen Verantwortung der Wissenschaft abzuleiten ist. Die Hochschulgeographie gibt ihren Studienabsolventen Vorstellungen über Welt und Umwelt mit, von denen diese sich dann z. B. als Planer bei konkreten Veränderungen dieser Umwelt leiten lassen oder die sie als Lehrer in das Bewußtsein der Schüler weitertragen und damit vervielfachen. In einem Fach, das in diesem Maße sowohl an der Bewußtseinsbildung als auch an der Lebensraumgestaltung beteiligt ist, sollte man sich darüber im klaren sein, daß bereits eine vermeintlich «reine Beschreibung» von Raumstrukturen gleichzeitig den Verflechtungsbereich Gesellschaft-Umwelt und dessen normative Grundlagen abbildet. Erst wenn diese in die Erklärung mit einbezogen und damit grundsätzlich zur Disposition gestellt werden, kann die Aufmerksamkeit nicht nur auf die Veränderungsmöglichkeiten, sondern auch auf die Verantwortung dafür gelenkt werden.

Der Öffentlichkeitsarbeit kommt aber noch eine andere Schlüsselstellung zu, und dies läßt sich besonders deutlich an solchen Themen wie der augenblicklichen Ökologiediskussion zeigen. Bisherige Warnungen von seiten derWissenschaft - etwa in bezug auf das «Waldsterben» oder andere Umweltgefährdungen - blieben vielleicht deshalb lange weitgehend unbeachtet, weil sie zu «steril» und wenig verständlich aufbereitet waren. Es ist natürlich schwer - angesichts der Schreckensmeldungen, die uns täglich erreichen und uns abstumpfen lassen -, die Öffentlichkeit wirklich «anzusprechen»; aber es ist notwendig, sie anzusprechen, denn Veränderungen können nicht in Gang kommen, wenn sie nicht auch in den Köpfen der Menschen stattfinden, und Umweltschutz z. B. wird nur greifen, wenn jeder aus Überzeugung aufgrund ausreichender Information bei sich selbst anfängt. Aussagen von Wissenschaftsseite können noch so richtig sein - solange sie im «Elfenbeinturm» gemacht werden und nur Zustimmung in der eigenen Wissenschaftsgemeinde finden, werden sie nichts verändern. Aber auch umgekehrt: selbst wenn sie bei anderen Wissenschaftlern auf Ablehnung stoßen - und das wird auch heute ebenso oft der Fall sein wie in zahlreichen Beispielen aus der Geschichte - können sie doch in einer sie tragenden Öffentlichkeit positive Veränderungen hervorrufen, wenn sie entsprechend allge- 
meinverständlich präsentiert und zur Diskussion gestellt werden. Ich meine, da $\beta$ die Geographie in der Lage ist, sehr viel zu einer solchen Diskussion beizutragen.

\section{Literatur}

BARTELS, D. (1968): zur wissenschaftstheoretischen Grundlegung einer Geographie des Menschen, in: Geographische Zeitschrift, Beiheft 19.

BLUNDEN, J. et. al. (Hrsg.) (1978): Fundamentals of Human Geography, London

BUTZIN, B. (1982): Elemente eines konfliktorientierten Basisentwurfs zur Geographie des Menschen, in: Sedlacek, P. (Hrsg.): Kultur-/Sozialgeographie, S. 93-124; Paderborn.

CAPRA, F. (1983): Wendezeit; Bern - München - Wien

DÜRR, H. (1979): Für eine offene Geographie, gegen eine Geographie im Elfenbeinturm, in: Karlsruher Manuskripte zur Mathematischen und Theoretischen Wirtschafts- und Sozialgeographie, H. 36 .

GALE, St. u. G. Olsson (Hrsg.) (1979): Philosophy in geography, Dordrecht.

GREGORY, D. (1978): Ideology, Science and Human Geography, London

HANTSCHEL, R. (1982): Räumliche Aspekte sozialphilosophischer Ansätze und Theorien, Frankfurt/M.

HANTSCHEL, R. (1985): Die Übertragung des systemanalytischen/-theoretischen Ansatzes auf räumliche System, in: Geographie und Schule, H. 33.

HARVEY, D. (1977): The Geography of Capitalist Accumulation: A Reconstruction of the Marxian Theory, in: Peet, R. (Hrsg.): Radical Geography, S. 263-292, London.

KLAUS, D. (1985): Allgemeine Grundlagen des systemtheoretischen Ansatzes, in: Geographie und Schule, H. 33.

LESER, H. (1975): Landschaftsökologie, Stuttgart.

LESER, H. (1980): Geographie, Braunschweig.

LEUZINGER, H. (1984): Wird die Geographie in den Medien vernachlässigt, in: Geographica Helvetica 1984, Nr.2, S. 107-109

LORENZEN, P. (1978): Theorie der technischen und praktischen Vernunft; Stuttgart.

OLSSON, G. (1977): Servitude and inequality in spatial planning: Ideology and methodology in conflict, in: Peet, R. (Hrsg.): Radical geography, S. 353-361, London.

PEET, R. (Hrsg.), (1977): Radical Geography: Alternative Viewpoints on Contemporary Social Issues, London.

ROSNAY, I. de (1977): Das Makroskop, Stuttgart.

SCHMIDT-WULFFEN, W. D. (1980): "Welfare Geography" oder: Leben in einer ungleichen Welt, in: Geographische Zeitschrift 1980, S. 107-119.

Schweizerische Gesellschaft für Angewandte Geographie (Hrsg.), 1985: Informationen in eigener Sache, Bern.

SEDLACEK, P. (1982): Kultur-/Sozialgeographie: Eine einführende Problemskizze, in: Sedlacek, P. (Hrsg.): Kultur-/Sozialgeographie, S. 9-22, Paderborn.

WIRTH, E. (1978): Zur wissenschaftstheoretischen Problematik der Länderkunde, in: Geographische Zeitschrift 66 (4), S. 241-261.
WIRTH, E. (1980): Die Blickverengung einer "offenen Geographie" und das Märchen vom Elfenbeinturm, in: Karlsruher Manuskripte zur Mathematischen und Theoretischen Wirtschafts- und Sozialgeographie, H. 41.

WÖHLKE, W. (1969): Kulturlandschaft als Funktion von Veränderlichem in: Geographische Rundschau, 21. Jg. H. 8.

\subsection{Claude Raffestin: De l'idéologie à l'utopie ou la pratique du géographe}

\section{L'évidence de l'objet}

Lequel, parmi ceux d'entre nous qui ont eu affaire avec une «intervention pratique» dans le territoire, n'a pas eu à surmonter l'incrédulité de ses interlocuteurs quant aux capacités d'un géographe à dire autre chose que l'évidence inscrite dans le paysage ou sa représentation? Dès lors, comment s'étonner que la géographie ait de la peine à s'insérer dans la pratique professionnelle alors qu'elle n'est pas jugée digne, dans la plupart des cas, de figurer dans le débat culturel en tant que porteuse de pensée réflexive? Pourquoi d'ailleurs en est-il ainsi? La question n'est pas rhétorique tant s'en faut!

Je crois qu'il en va ainsi par la faute même des géographes qui, à peu de choses près, renvoient l'image que les représentants des autres disciplines se font d'eux: «l'image d'un quelque chose entre ciel et terre». Les géographes se sont enfermés dans l'évidence de l'objet. En effet, à force de définir leur objet par ce qui est donné ou offert à l'observation et à la description entre «ciel et terre», les géographes sont assiégés par le paradoxe de l'évidence. Si la géographie est une construction à partir d'un ou plusieurs appareils conceptuels, il est paradoxal que l'objet lui-même ne soit pas construit: ou bien l'ensemble est construit ou bien la géographie n'est pas une construction et alors elle n'existe pas en tant que discipline.

L'objet offert, «ce quelque chose entre ciel et terre», ne saurait être l'objet géographique mais la matière première à partir de laquelle celui-là peut être construit. Si le géographe a tellement de peine à s'insérer dans une pratique, c'est essentiellement parce qu'il part d'une évidence qu'il soumet à des procédures diverses au lieu de construire un objet théorique à partir duquel il pourrait générer des pratiques. En d'autres termes, la pratique du «quelque chose entre ciel et terre» sur laquelle je reviendrai, n'a pas été le fondement pour l'invention d'idéalités géographiques. On pourrait ici faire une analogie 
avec la géométrie. L'arpentage, comme l'a montré Husserl, a été le fondement pour la grande invention de l'idéalisation géométrique. Ce qui aurait pu être le fondement de la géographie, à savoir le voyage, n'a que très imparfaitement joué son rôle, et cela justement parce que le voyage n'a pas été pensé comme donateur de sens à une idéalisation mais comme créateur de sécurité pour la mobilité future des hommes (DEMATTEIS, p. 16).

Le voyage, toujours présent pour les géographes, a été rebaptisé travail sur le terrain mais avec la même ambiguïté de l'évidence de l'objet. Si le «quelque chose entre ciel et terre» est donateur de sens, il n'est pas l'objet géographique mais pré-géographique. Dans ces conditions, la géographie a longtemps tourné autour d'une triade: repérer, localiser, identifier des objets dont la représentation semble avoir été longtemps plus cartographique que géographique. Or tout notre enseignement, même si cela change, a longtemps tourné autour de cette triade qui s'est révélée dans le passé parfaitement suffisante mais qui ne l'est plus pour féconder une pratique. Cette triade classique est d'autant plus insuffisante qu'elle a été disqualifiée par la télédétection qui peut assumer ces fonctions pratiquement en temps réel. Curieusement c'est l'apparition d'un ensemble de techniques qui nous contraint aujourd'hui à construire des idéalités géographiques si nous ne voulons pas voir disparaître notre discipline.

Ce qui faisait hier notre spécificité n'est plus aujourd'hui que banalité que les techniques restituent plus précisément que nous ne saurions le faire avec nos moyens classiques. Néanmoins, c'est certainement la chance que nous devons saisir car ces instruments peuvent permettre de créer une véritable géographie en nous déchargeant de travaux dont la réalisation est automatisable.

Qu'est-ce à dire? Que le projet social de la géographie, autrement dit son idéologie, est en train de changer.

\section{Le décalage idéologique}

La géographie a longtemps vécu sur le projet de représenter «objet évident» par la carte. Celle-ci est devenue l'objectif à réaliser. De la carte topographique à la carte thématique, on a assisté à une variation sur le même thème qu'on est loin d'avoir épuisé si l'on en juge par la floraison d'Atlas en tous genres qui sont actuellement publiés. Pourtant, dans cette perspective, on se trompe car on prend le moyen pour la fin. La carte n'est qu'un moyen, un instrument comme d'autres au service de la géographie, elle ne saurait être une fin.

Pourquoi? Parce que la cartographie s'intéresse à la consignation des choses. Or le monde auquel s'inté- resse la géographie n'est pas l'ensemble des choses mais l'ensemble des faits. Or un fait est l'existence d'états de choses et l'état de choses est une liaison de choses. L'objectif de la géographie est la liaison, la relation entre les choses.

Le nouveau projet social de la géographie, celui-là même qui est postulé par les nécessités d'une pratique spatiale et territoriale, est constitué par les liaisons qui existent entre les états de choses. Mais, me direz-vous, c'est bien à cela que nous nous intéressons, c'est bien cela que nous nous efforçons de faire. Ce à quoi je vous répondrai que c'est imparfaitement le cas. En effet, l'absence d'une géographie, aus sens où il existe une géométrie, se ressent différentiellement selon les échelles. Aux petites échelles, l'accent est certainement mis sur les liaisons et les relations, aux moyennes échelles c'est déjà moins vrai et aux grandes échelles ce n'est plus vrai du tout. Nous avons été attentifs au macro, un peu moins au méso, plus du tout au micro. Tout notre enseignement est basé sur le macro et le méso mais peu sur le micro qui est justement le lieu d'intervention privilégié des praticiens. Les géographes parviennent mal à faire le transfert de la réflexion géographique du niveau macro au niveau micro. Notre discipline lorsqu'elle est confrontée au micro se dissout et il ne nous reste dans les mains que fort peu de choses sinon des instruments empruntés à la cartographie ou à l'économie. De véritable réflexion géographique aux grandes échelles, il n'y en a pas ou fort peu. Je dis bien de véritable réflexion géographique qu'il ne faut pas confondre avec l'application de méthodes statistiques par exemple.

Que demande-t-on à un géographe engagé dans la réalisation d'un plan directeur de commune par exemple? D'être capable tout à la fois de repérer les liaisons entre les choses et de prévoir l'évolution de ces liaisons ou plus modestement d'esquisser les évolutions possibles de ces liaisons. Cela nous ne le faisons encore que très mal et c'est sans doute pourquoi nous avons tellement de peine à nous imposer en tant que praticiens. Nous savons décrire un système territorial communal mais nous ne savons pas saisir une écogenèse territoriale et son mouvement vers le futur.

Cela revient à dire que les difficultés que nous rencontrons sont la conséquence d'un décalage idéologique. L'idéologie étant prise ici dans le sens de projet social. L'ancien projet social de la géographie est complètement obsolescent et oblitéré. Il a suffi pendant longtemps à nos besoins qui ne demandaient pas autre chose que l'ancienne triade appliquée aux objets spatiaux. Mais cela ne suffit plus puisqu'il faut être en mesure de substituer aux choses les états de choses en tant que ceux-ci sont porteurs de liaisons et de relations.

Le nouveau projet social de la géographie doit s'enraciner dans la construction d'idéalités géographiques qui permettent d'aborder la grande échelle. Il 
ne s'agit nullement de s'éloigner de la pratique du voyage, c'est-à-dire du terrain, bien au contraire mais de retourner à l'objet évident donateur de sens sur lequel peuvent et doivent être construites ces idéalités géographiques à propos desquelles j'aimerais donner quelques éclaircissements.

Tout plan directeur est un système de limites mais que savent dire les géographes sur les limites? Les principes d'aménagement invoquent des centres, des liaisons, des axes mais là encore que savonsnous de ces notions au niveau micro? Quand je dis que «savons-nous», j'entends que savons-nous réellement au-delà du sens commun qui les sature à l'envi? Si peu de choses que dernièrement alors que nous discutions à la Commission d'urbanisme d'un centre, les architectes et les urbanistes ont polémiqué durant deux heures pour savoir si ce qui était proposé était oui ou non un centre! Ce n'était pas leur faute mais la nôtre car nous ne leur avons pas fourni un concept utilisable de centre.

J'ai vécu la même expérience il y a dix jours à Québec à propos de la notion de capitale. Au-delà de la définition du dictionnaire et de rares informations de géographie urbaine, personne ne savait réellement ce qu'était une capitale. Prétendra-t-on encore que nous ne sommes pas victimes de l'évidence de l'objet?

Lorsque la géographie humaine aura appris à construire les objets sur lesquels elle travaille, elle sera en mesure de jouer un rôle pratique réellement efficace. Cette construction constituera, l'emploi du futur signifie que nous y parviendrons, d'ailleurs un puissant moyen d'intégration des différentes parties, aujourd'hui encore dispersées, de la géographie. Les notions de limite et de centre pour demeurer à l'intérieur de ces deux exemples n'appartiennent pas comme on pourrait le croire traditionnellement à la géographie politique et à la géographie urbaine mais à toutes les branches de la géographie. Les notions de limite et de centre sont trans-géographiques car on les retrouve et on en a besoin dans absolument tous les domaines spécialisés de la géographie. Mais il ne saurait y avoir de construction sans retour au «sol du monde immédiatement perçu» pour reprendre une idée-force de Husserl.

Mais attention, il ne s'agit pas d'une géométrisation de la géographie lorsque j'évoque les notions de centre et de limite parmi beaucoup d'autres. La construction des objets géographiques en tant qu'états de choses, c'est-à-dire de faits qui arrivent comme dirait Wittgenstein, nécessite une nouvelle idéologie, un nouveau projet qui s'enracine dans l'histoire pour comprendre l'écogenèse territoriale et les pratiques que les hommes ont eu de l'espace mais aussi dans l'utopie pour suivre les aspirations collectives. L'utopie, le mot est lâché, si mal comprise qu'un fonctionnaire fédéral me disait, il y a peu de temps, que c'était un luxe que nous ne pouvions pas nous payer. Je crois qu'il est possible de renverser sa formule car au contraire, c'est le nécessaire que nous ne savons pas nous payer malgré notre richesse. C'est même l'indispensable qui fait cruellement défaut dans notre société. L'absence de dimension utopique donne des sociétés sans projets, sans espérance et partant souvent profondément désespérées. Un professeur de médecine préventive et sociale me disait il y a quelque temps que le nombre des suicides en Suisse était au même niveau que les accidents mortels. Il y a là matière à réflexion.

\section{De l'utopie à la prospective}

L'ancienne idéologie de la géographie est complètement réalisée ce qui est paradoxal selon la pensée de Mannheim pour qui une idéologie est et demeure toujours incomplètement réalisée. La géographie se cherche un nouvel horizon, un nouveau projet au risque de disparaître si elle ne le trouve pas.

Si nous n'avons pas réussi à élaborer une réelle pensée géographique à partir de l'évidence de l'objet, alors il faut renverser le processus en pensant l'objet à réaliser. Il faut introduire la dimension utopique sous la forme d'une réflexion prospective. Penser le territoire à créer c'est se contraindre à construire un objet géographique imaginaire cohérent et adapté à l'espace donné. En partant de la "parole territoriale», nous ne sommes pas parvenus aux idéalités géographiques. Peut-être qu'alors en construisant une «langue territoriale» et en retournant à la parole parviendrons-nous à comprendre les liaisons et les relations contenues dans les états de choses.

Pourquoi cette dimension utopique est-elle nécessaire? Parce que de plus en plus, on demande au géographe confronté aux réalisations pratiques d'être en mesure d'évaluer les tendances et les effets les plus plausibles dans le domaine territorial. Dès lors, cela signifie que nous devons être capables de déterminer un futur probable en établissant une échelle probabiliste des permanences. Mais nous devons aussi être capables de délimiter le champ de futurs possibles et le rôle de certaines transformations. De même, il faut savoir imaginer des territoires futurs désirables ou souhaitables cohérents avec les conditions des écosystèmes.

Finalement, il faut savoir créer des utopies apprivoisées et cohérentes alors que les utopies classiques unissent la science, la critique et la fantaisie. Je ne veux pas dire qu'il faut bannir la fantaisie mais qu'elle ne saurait être le résultat d'une imagination débridée et incohérente.

Cela implique des conséquences pour notre enseignement universitaire. Au lieu d'étudier la géographie uniquement dans ses réalisations actuelles, il faut la concevoir comme une structure à modifier et qu'on modifiera si certaines conditions économiques, techniques, sociales et culturelles se réalisent. 
Les géostructures que nous avons sous les yeux sont des produits d'un passé et évoluent vers un futur inconnu ou mal connu. Dès lors, il faut penser le territoire non pas seulement sur l'axe passé-présent-futur mais aussi dans l'autre sens futur-présent-passé pour en avoir une connaissance à peu près satisfaisante.

Par ailleurs, il faut introduire dans cette réflexion perspective des accidents: crise, catastrophes, modifications progressives ou brutales et chercher à expliciter les effets sur la géostructure et surtout sur les relations à l'intérieur de la géostructure. Cela suppose de concevoir les états de choses en réseaux et de confronter ces images construites aux réalités géographiques donatrices de sens.

Bien comprendre une géostructure actuelle c'est se demander ce qu'elle peut devenir sous l'influence de certains facteurs à terme. La géographie est soustendue par une discipline qui est à créer mais qu'on pourrait déjà nommer la diathétique et qui rendrait compte des arrangements spatiaux. La diathétique à créer est peut-être l'un des moyens pour construire l'objet géographique: arrangements conditionnés par des principes ordonnateurs conscients ou assimilés par une pratique répétitive. La diathétique pourrait être pourvoyeuse d'idéalités géographiques. Il faut pour cela changer notre conception de l'enseignement de la géographie, partir d'éléments de la pratique que les hommes ont de l'espace et faire jouer avec des dispositions. Les géographes ne jouent pas assez avec l'espace au contraire des praticiens architectes et urbanistes qui ne cessent de jouer un jeu réel et dangereux parce qu'impliquant du vivant. C'est parce que les géographes ne jouent pas assez qu'ils ne sont généralement pas en mesure de dénoncer le caractère dangereux des jeux réels.

Il ne s'agit pas de «jouer» mais d'introduire une dimension ludique pour faire bouger la pensée géographique. En matière d'aménagement cette dimension ludique est indispensable. Deux exemples suffiront. Si le raccordement Praille-Eaux-Vives avait été réalisé dans les années 50 , le problème de la circulation à Genève serait en grande partie résolu. Or, on y pense seulement maintenant. Les autorités hésitent à soutenir le projet de canalisation du Rhône de Lyon à Genève et vraisemblablement elles renonceront. Sur le plan d'une politique de l'environnement, elles ont tort. L'évolution des projets en termes strictement économiques est devenue insuffisante et il faut lui ajouter une évaluation en termes d'environnement car les problèmes majeurs d'ici 15 ans seront environnementaux. C'est pourquoi les géographes doivent s'y préparer.

\section{La pratique du géographe entre idéologie et utopie}

La géographie fondée sur une idéologie complètement réalisée et refusant encore l'utopie est en train de se couper de possibilités d'interventions pratiques. Toutes les disciplines sollicitées par la pratique se déplacent sans cesse entre les deux pôles idéologie et utopie. Cette mobilité est le seul moyen de préserver les capacités innovatrices d'une discipline. L'axe idéologie-utopie est un axe de circulation des idées et donc des innovations.

Cette mobilité entre idéologie et utopie semble être une nécessité pour élaborer une véritable réflexion géographique susceptible de fonctionner à toutes les échelles. Jusqu'à maintenant, la géographie s'est développée sur un axe sémantique et ce dont nous avons besoin pour la pratique c'est d'un axe syntaxique: autrement dit à une logique des fonctions, il faut substituer une logique structurale qui fera apparaître les relations.

Pour nous insérer dans la pratique, la logique structurale du code syntaxique peut seule permettre par combinaisons successives de s'ouvrir vers la prospective. En demeurer au code sémantique, c'est rester attaché à l'idéologie.

La condition de la reussite d'une pratique est l'existence d'une théorie fondée sur une logique structurale. C'est d'autant plus vrai que dans le cas contraire on passe d'un cas particulier à un autre et qu'on juge d'une manière arbitraire. Il suffit d'avoir fonctionné dans un service d'aménagement du territoire pour le savoir. L'incapacité dans laquelle on est pour décider provient, entre autres, de l'absence d'une véritable dénotation structurale.

\subsection{Charles Hussy: Aspects internes et externes d'une identité}

Quand on m'a proposé de m'intégrer aux groupes de travail «identité» qui couvrait la Suisse romande, l'idée a surgi très vite dans mon esprit qu'une image "propre» ou interne (ou Selbstbild) devait se doubler d'un regard extérieur (ou Aussenbild) sur la géographie et les géographes. Or, qui pouvait mieux témoigner à cet égard que le monde du travail, à travers, d'ailleurs, géographes et non-géographes placés en situation d'employeurs pour des géographes? D'où cette enquête, dont les résultats seront l'essentiel de mon propos. Ils n'ont pas été diffusés car ils recouvrent un domaine (la pratique des géographes) dont s'est occupé le groupe de Berne. Ils pourront l'être par la suite. Ici déjà, la relation pourra être faite entre les éléments professionnels d'évaluation que je vais vous livrer et les impressions sub- 
jectives qui ressortent de l'enquête auprès des géographes; je prends immédiatement un exemple. On a vu que les géographes dont l'activité se situe dans les champs professionnels non-classiques (tels que l'emploi dans un service de planification ou dans une entreprise) sont très mobiles et ressentent une insécurité; on ne s'en étonnera pas, quand on saura que les employeurs expriment les plus grandes réserves quant à la préparation des étudiants à une insertion dans la praxis. Un géographe, aujourd'hui, n'entre guère dans le marché privé qu'au prix d'une certaine perte d'identité. "Il est géographe parfait, dira-t-on, ce n'est pas une tare, mais voyons ce qu'il sait faire?» Voilà le choc de la réalité de notre image du-dehors. On n'est plus au temps où Jean Gottmann tenait des propos du genre: «Le géographe enrichit la connaissance scientifique de notre milieu et de notre époque dans des domaines divers»; ou encore, disait Jean Gottmann, «Le géographe est profondément conscient de la complexité des problèmes régionaux» ou encore «L'étude de la planification régionale, conçue et conduite dans un esprit géographique (...) devrait éliminer des erreurs dues à trop d'empirisme dans la planification et son application.» Ces déclarations datent de 1950 et depuis lors, qu'a-t-on fait? On est demeuré victime d'une évidence de l'objet, nous a dit Claude Raffestin. Avons-nous appris, comme l'a écrit Braudel, à «interroger la société à partir de l'espace»? En France il existe une forte demande de géographes qui n'est pas satisfaite, de l'avis même des professionnels et de groupes associatifs. Il n'en va pas de même en Suisse, mais, comme en France, on y recrute des économistes, des sociologues, on confie l'aménagement à des architectes urbanistes, faute de relations entre utilisateurs de géographie et géographes, mais aussi faute de relations entre théoriciens et praticiens de la géographie.

Voyons donc où en est notre image dans des milieux concernés non seulement par la préparation de praticiens géographes, mais à la source, par une meilleure connaissance de la géographie, de son champ de validité, de son utilité sociale.

\section{Résultats du sondage auprès d'organismes publics ou privés susceptibles d'engager des géographes}

L'échantillonnage visait pour moitié des offices ou administrations publics, pour deux cinquièmes environ des bureaux privés d'aménagement ou de planification et, enfin, dans trois cas sur dix, des entreprises privées. Les réponses récoltées, au nombre de 27 sur un total prévu de 50 , se ventilent sensiblement de la même manière. On a effectivement 14 organismes publics ou d'intérêt public, mais 7 bureaux privés et 6 entreprises. Précisons d'emblée que plus de la moitié (soit 16 cas) recrutent des géographes; ceux-ci procèdent, en général, à une évaluation plus positive que les autres à l'endroit des géographes. En second lieu, la plupart des personnes interrogées sont de formation universitaire et là encore, on constate de leur part une évaluation plus positive des géographes, en même temps qu'une évaluation moins positive, en revanche, des universitaires. Les interrogés sans formation universitaire montrent une tendance à idéaliser dans l'abstrait l'universitaire en général.

L'universitaire est également mieux perçu par les entreprises que par les organismes publics, le contraire étant vrai pour les géographes, mieux appréciés de la part du secteur public et ce phénomène semble assez peu en accord avec l'expérience en matière de politique de la recherche en Suisse. Autrement dit, si les géographes ont de la peine à se faire reconnaître comme des collaborateurs valables dans le cadre des programmes nationaux de recherches, dans le secteur des bureaux d'étude - concurrents des instituts universitaires - et des organismes publics ou semi-publics, ils trouvent une bonne audience et des débouchés.

En tout et pour tout, nous disposons d'interviews (Tableau 1) d'une douzaine offices publics, dont la moitié n'utilisent pas les services d'un géographe (parmi eux, les offices de protection de l'environnement de la Confédération et du canton de Berne), de 7 bureaux privés dont 5 en occupent un, voire même plusieurs (CEAT, Urbaplan, Scope-Lucerne ou le Bureau Berz et Droux à Berne, par exemple). Parmi les 6 entreprises privées de notre échantillon, seul Kümmerly \& Frey emploie deux géographes; et cependant certaines se disent prêtes à envisager sérieusement une candidature de géographe: pour Firmenich (Genève) il n'existe aucune contre-indication au départ, à condition, ajoute Dupont-de-Nemours, que l'universitaire formé dans une discipline accepte de travailler dans un domaine qui n'est pas le sien au départ. Par antithèse, la Vaudoise-Assurances avoue qu'à ses yeux, "ce serait là un vrai luxe» et Nestlé préfère engager des commerciaux, tandis qu'Hotelplan affirme avoir fait une mauvaise expérience en plaçant dans son secteur des réservations divers étudiants géographes.

La participation à cette enquête est évidemment fonction des collaborations trouvées dans les différentes villes de Suisse, et aussi des habitudes mentales des employeurs. L'attitude de certains organismes ne laisse pas d'étonner, comme le refus de l'interview par le Bureau International du Travail, qui affirme ne pas se sentir concerné par la question.

Cet échantillon, faible mais à mes yeux représentatif, est donc censé fournir des éléments de l'image que véhicule de nous, géographes, le milieu des employeurs. Mon intention n'est pas de livrer ici une profusion de chiffres, néanmoins les résultats de cette consultation m'ont paru suffisamment clairs pour évoquer devant vous trois apects de notre 
image au sein des milieux concernés par l'engagement professionnel des géographes. Mon exposé s'articulera donc en trois parties, que je baptiserai lapidairement "portrait-robot", "sphères de compétence» et "perspectives d'engagement». En effet, ce seront d'abord les qualités retenues pour caractériser l'universitaire en général et le géographe en particulier, sorte de portrait-robot obtenu par le traitement de questions closes. Ensuite et en second lieu, nous parlerons d'interdisciplinarité, c'est-à-dire que nous examinerons dans quels domaines est reconnue aux géographes une capacité de franchir les limites de leur discipline et, pour conclure, nous noterons en substance les idées et suggestions émises selon les catégories d'interrogés établies par l'analyse, de façon à pouvoir conclure sur un ton plutôt qualitatif et restituer un certain nombre de suggestions individuelles.

\section{Tableau 1. Liste des organisations interrogées}

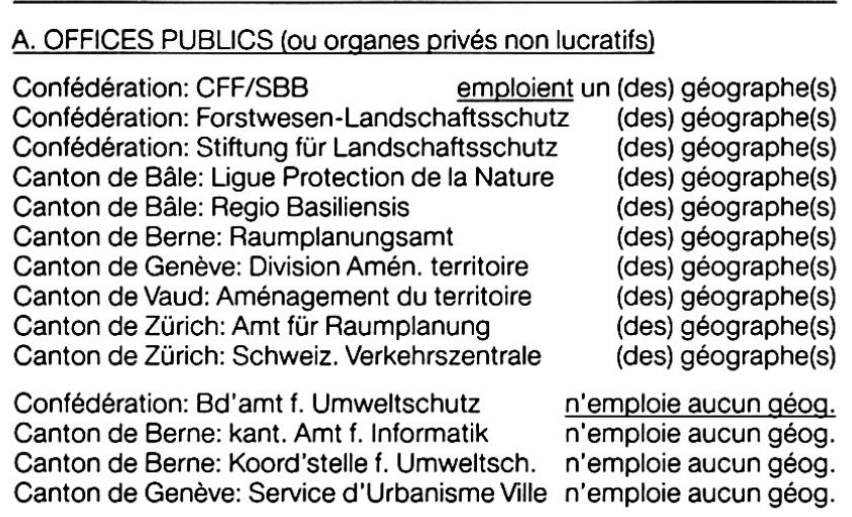

\section{B. BUREAUX PRIVÉS, CENTRES DE RECHERCHE, etc.}

Niv. suisse: Schweiz. Fremdenverkehrsverb. Canton de Berne: Berz-Droux Planung Cant. de Luc.: DemoSCOPE, Markforsch'inst.

Canton de Vaud: CEAT Lausanne

Canton de Vaud: Urbaplan, am. terr.

emploie un (des) géog. emploie un (des) géog. emploie un (des) géog. emploie un (des) géog. emploie un (des) géog.

Canton de Berne: Vatter-Hebeisen, Planung Canton de Genève: Centre de Rech. Battelle

n'emploie aucun géog. n'emploie aucun géog.

\section{ENTREPRISES PRIVÉES}

Canton de Berne: Kümmerly \& Frey

Canton de Genève: Dupont de Nemours Canton de Genève: Firmenich, ind. chimique Canton de Vaud: Nestlé (Vevey)

Canton de Vaud: Vaudoises Assurances Canton de Zürich: Hotelplan, voyages emploie un (des) géog. n'emploie aucun géog. n'emploie aucun géog. n'emploie aucun géog. n'emploie aucun géog. n'emploie aucun géog.

\section{Les attributs de «l»'universitaire et «du» géographe}

J'ai analysé en valeur relative les réponses selon sept critères d'évaluation proposés, qui sont, pour les résumer en peu de mots, l'analyse, la synthèse, l'autonomie, la créativité, la méthodologie, l'esprit de collaboration et le sens des réalités. Les interrogés devaient affecter à chaque rubrique une mention «peu important», «moyennement important» out «très important». Ces notations se regroupent en quelques composantes principales, puisque on les retrouve associés sur trois axes factoriels qui saturent respectivement $43 \%, 27 \%$ et $21 \%$ de la variance. Voici ces trois facteurs:

Tableau 2. Regroupement factoriel des qualités reconnues aux géographes

FACTEUR 1 (43\%) Aptitudes essentiellement personnelles:

- “Esprit de CRÉATIVITÉ, faculté d'imagination» (universitaires et géographes)

- “SENS DES RÉALITÉS pratiques, des limites d'efficacité propres à toute planification" (univ. et géog.)

- "Sens de la COLLABORATION et du dialogue" (géographes seulement)

Un peu mieux ressenti dans l'entreprise privée et chez ceux qui emploient des géographes. C'est ainsi que réagissent Pro Natura ou l'Office d'Aménagement du Canton de Berne, qui comptent plusieurs géographes et disent accorder autant d'importance à la personnalité qu'à la formation.

FACTEUR 2 (27\%) Contenu "académique" de la formation:

- "Hauteur de vue, aptitude à I'ANALYSE de situations" (univ., géog.)

- "Esprit de SYNTHĖSE, capacité d'articuler des systèmes" (idem)

Mieux ressenti par ceux qui emploient des géographes, notamment Regio Basiliensis et les CFF.

FACTEUR $3(21 \%)$ Qualités professionnelles:

- "AUTONOMIE dans la recherche, sens des responabilités" (id.)

- "Connaissance et maîtrise d'une METHODOLOGIE" (id.) Souligné davantage par des non-universitaires. Certains offices d'aménagement publics, Battelle, Nestlé, etc.... insistent avant tout sur l'expérience, voire la specialisation.

La meilleure convergence d'opinion va donc aux traits d'évaluation personnelle et le moins bon accord se fait sur les qualités plutôt professionnelles des universitaires et des géographes. L'étoffe personnelle est primordiale aux yeux, notamment, du secteur privé et elle est largement reconnue aux géographes par les offices publics faisant appel à leurs services. C'est d'ailleurs l'idée que l'on retrouvera dans les remarques du type: "La personnalité, le caractère conditionnel la moitié des débouchés» ou «C'est à son propre esprit d'initiative, et non à la formation universitaire, que l'on doit d'être ou non un bon praticien.» 


\section{Compétences interdisciplinaires}

L'ordre décroissant des domaines concédés aux géographes est le suivant (d'après le cumul compensé des «oui» et des «non»):

Tableau 3. Attribution de compétences particulières sur des questions touchant à:

\begin{tabular}{|c|c|c|}
\hline Aménagement du territoire & score net & $22 / 27$ soit 0.9 \\
\hline Cartographie & & $20 / 22$ soit 0.9 \\
\hline Statistique & & $16 / 18$ soit 0.9 \\
\hline Géologie & & $17 / 21$ soit 0.8 \\
\hline Problèmes d'impact (env.) & & $19 / 25$ soit 0.8 \\
\hline Sociologie & & $15 / 20$ soit 0.7 \\
\hline
\end{tabular}

Techniques graphiques

$4 / 18$ soit 0.22

Anthropologie

Psychologie

Science politique

0: jusqu'ici plutôt "Oui"

$-2 / 18$ soit -0.1 : «non" $-5 / 20$ soit -0.2

Marketing

$-14 / 23$ soit -0.6

La compétence moyenne (tous critères pris ensemble) est évaluée plus sévèrement par les offices publics $(0.3)$ que par les bureaux privés $(0.4)$ et les entreprises (0.5), et cela même dans le domaine de l'aménagement du territoire. Pareillement, le crédit relatif consenti au géographe dans les questions d'impact, de risque et de contrainte naturelle est davantage le fait des entreprises que d'offices spécialisés. Mais faute de savoir de quoi au juste s'occupe la géographie et donc, à quoi peuvent servir les géographes, l'entreprise lui reconnaît peu de compétence sur le terrain pratique (du marketing, notamment). Je crois que cette question fournit un élément essentiel d'information par rapport à l'image:

\section{$I M A G E$ "INTERNE» = SPÉCIFITÉ du géographe}

Certaines personnes interrogées - elles-mêmes géographes - ont indiqué d'autres spécialisations telles que la climatologie, la démographie ou l'étude rurale, ou encore le «sens du terrain». Si unanimité il y a à propos de cette question, c'est pour affirmer que la géographie devrait se créer, s'inventer elle-même une spécificité, ou alors définir mieux son domaine propre et ses outils propres. Ceux qui disent cela avec véhémence se préoccupent bien évidemment de la formation de praticiens.

\section{IMAGE «EXTERNE» = un GENEERALISTE compétent}

Car un autre avis, minoritaire mais émanant de l'une ou l'autre grande firme industrielle, dénie au géographe toute spécificité: qu'il soit généraliste et perde ses complexes d'inutilité, qu'il s'assure pour la vie professionnelle la maîtrise d'une langue, qu'il «soit géographe mais sache offrir autre chose». Le grand tort des géographes, c'est de vouloir vendre ce qu'ils aiment et non ce qui intéresse les autres, autrement dit de chercher à s'imposer et non à être efficients sur le plan pratique. Ce type de licencié souffre d'un taux de dispersion intellectuelle supérieur à la moyen$n e$, à l'heure même où des questions qui le concernent au premier chef (l'aménagement de l'espace) prennent une acuité toujours plus grande. Être un généraliste, c'est être sensible aux multiples aspects d'un problème, ce n'est pas être dispersé dans sa connaissance.

\section{L'analyse par tendances des perspectives professionnelles et l'image}

L'image de la géographie elle-même est floue, et c'est d'ailleurs principalement celle de la géographie scolaire. L'image du géographe, elle, est parfois d'une inquiétante acuité. Ainsi cette assistante au personnel d'une grande agence de voyages n'hésite-t-elle pas à déclarer: «Die Geographen brauchen mehr Realitätssinn, Integrationsfähigkeit, Integrationswille, Arbeitsdisziplin. Geographen sind Träumer.»

L'abondance des annotations personnelles m'interdit de restituer sans système une image de la discipline et de ses problèmes. J'ai donc repris ma première analyse et regroupé les interrogés selon les scores des facteurs, secondairement selon le type d'organisme. Quatre groupes se dégagent, qu'il faudra considérer comme quatre optiques différentes quant au profil personnel, intellectuel et professionnel du géographe. Tout en résumant les avis et en cumulant les observations convergentes, je citerai au passage les réactions les plus saillantes et les plus significatives; il me semble toutefois préférable, à ce stade, de respecter l'anonymat des prises de position.

Tableau 4. Angle de vision des perspectives pour les géographes.

a) Organismes insistant sur les qualités personnelles (facteur 1)

Bd.amt Landschaftsschutz, Umweltschutz; Berne: Informatik, Umweltschutz, Genève: Am. du territoire, Urbanisme Ville; Vaud: CEAT, Vaudoise Assurances.

«Heute grosser Einsatzbereich»: le mot «engagement» retentit dans ce groupe comme un défi et comme un appel. Quel rôle professionnel peut jouer un géographe, quels sont ses outils en dehors de son légendaire «sens du terrain»? L'homme de la carte n'impressionne plus guère, et l'architecte, par exemple, dispose d'une approche plus visuelle de 
l'urbain. Ainsi, la formation géographique est-elle en perte d'image, et d'ailleurs l'offre universitaire en général est fort méconnue actuellement: "Die reine Forschung wird heute absolut nicht mehr gefragt» et le «bon» géographe est celui qui sait non seulement proposer une problématique du territoire, mais qui sait, de par ses qualités personnelles mieux que par ses études, étayer sa présentation.

En résumé: personnalité, créativité, réalisme, sens de la collaboration conditionnent l'utilité sociale du géographe.

b) Organismes insistant sur les qualités intellectuelles (facteur 2)

CFF, Centrale Suisse du Tourisme, Battelle, bureau BerzDroux, Demoscope, Urbaplan, Kümmerly \& Frey.

Demain, les généralistes sauront mieux se «vendre»; un bon planificateur doit avoir l'esprit de système. Or, il apparît que la géographie universitaire manque gravement à sa mission formatrice. D'une part, la géographie théorique est triste, notamment par une sorte de mentalité antipratique et l'absence de créativité en géographie appliquée. Ensuite, l'ossature universitaire de la géographie en Suisse est débi$l e$, fractionnée en unités régionales souvent trop petites et qui, en général, se comportent en tours d'ivoire, coupées du contact entre elles mais aussi, avec le planificateur. «La plupart des professeurs d'université, nous dit-on, n'ont pas d'expérience de la pratique; au contraire, quelqu'un qui aimerait aller voir ailleurs est pénalisé; sa carrière universitaire est finie. (...) Les profs de géographie sont en général des gens qui n'aiment pas voyager (...) Être interdisciplinaire veut dire qu'on sache juger quelle facette d'un problème mérite le plus d'attention.» Selon d'autres interlocuteurs, considérer le géographe comme un "diplômé» est une erreur: c'est un licencié d'université qui n'a à offrir - tout au moins on l'espère - qu'une autonomie de pensée, une compétence académique, qu'on voudrait voir mieux étoffée dans les domaines démostatistiques, écologique, etc. La spécificité du géographe devrait être une connaissance profonde et large du monde physique et social.

En résumé: le cloisonnement intra-universitaire (théoriciens, praticiens) et inter-universitaire appauvrit l'éventail de la formation.

c) Organismes insistant sur la méthodologie.

le metier (facteur 3)

Schweiz. Stiftung f. Landschaftsschutz, Schweiz Fremdenverkehrsverband; Berne: Raumplanung; Basel: Pro Natura, Regio Basiliensis; Zürich: Raumplanung; Vaud: Am. Territoire
La géographie a beaucoup contribué à forger l'opinion jusqu'à nos jours, et maintenant elle doit mieux se définir. A travers ceux qui la font, elle manque d'intelligence pratique et d'efficience. Il est absurde de donner une même formation à de futurs enseignants du niveau secondaire et aux futurs géographes «appliqués». Pour faire de la géographie un métier, il faut instaurer une POSTFORMATION: il est urgent d'apprendre aux gradués à intégrer connaissance et praxis, et à établir les bases de toute planification. Une certification postlicence pourrait former des généralistes à forte spécificité (personnalité), en facilitant le contact à la pratique en cours d'études. C'est donc la fin de la formation qui fait défaut et qui explique une dispersion supérieure à la moyenne quant à la qualité des licenciés. Bien peu sont prêts à la collaboration, bien peu maîtrisent, audelà des méthodes scientifiques d'analyse, une méthodologie du travail et de la gestion. Les géographes de profession n'ont pas encore ce «plus» à offrir, sans parler des aspects «artisanaux» de la formation: les langues, l'informatique, des rudiments de droit...

Logiquement donc, ce groupe qui valorise les méthodes et l'autonomie fait un procès sévère à l'enseignement universitaire qui n'a rien à offrir, selon lui - en matière, par exemple, des scénarios d'impact - au-delà des troncs communs de licence.

En résumé: absence de POSTFORMATION, cause d'incompétence

\section{d) Entreprises (n'ayant pas de géographes)}

pondérant le même facteur

Berne: Vatter-Hebeisen (Archit., Planung); Genève: Firmenich, Dupont-de-Nemours; Vaud: Nestlé; Zürich: Hotelplan.

L'entreprise demande aux universitaires une flexibilité; leurs chances d'emploi augmentent car, de nos jours, une bascule s'opère dans le sens des généralistes et de la mobilité intra-entreprise. Un géographe est donc jugé «utilisable même en marketing, ou en tout poste à responsabilité, s'il s'intègre». En fait, ceci relève de la musique d'avenir. Actuellement, les grandes firmes font davantage confiance à des spécialistes, par exemple aux ingénieurs de l'environnement. Reprenant sans le savoirVidal de la Blache, on pense que les géographes connaissent les lieux, mais non les hommes, que leur formation est trop vaste pour leur ouvrir de larges débouchés dans le secteur privé. Il n'y entreront guère qu'à titre individuel, ayant acquis une expérience du travail et la maîtrise des langues, ayant aussi perdu leurs complexes d'académisme et de science poussiéreuse.

En résumé: pour s'imposer dans l'entreprise, faire preuve de flexibilité 


\section{Conclusion}

Les organismes interrogés dans cette enquête sont, dans l'absolu, peu nombreux mais ils représentent un large éventail d'employeurs actuels ou potentiels. Certes, il faut regretter quelques grands absents, tels que l'ORL du Poly de Zürich ou les services d'aménagement de Fribourg ou de Neuchâtel, qui avaient été prévus dans l'échantillon mais la coordination avec les enquêteurs prévus n'a pas fonctionné; les entreprises, notamment du tertiaire, les multinationales sont également peu nombreuses. Les résultats auraient-ils été fort différents? Je ne pense pas, car les avis de trois ou quatre sociétés ou organes d'une même catégorie finissent par se recouper. Nous obtenons quelques idées suffisamment évocatrices, voir provoquantes comme celle qu'un géographe français, $M$. Allefresde résumait fort bien au Géoforum de Lyon en 1984: d'après lui, se demander quelle géographie appliquée on doit enseigner constitue une fausse question: «La géographie appliquée n'existe pas, soulignait-il, mais il existe mille façons d'appliquer la géographie.»

\subsection{A) K. Aerni: Schweizer Geographie am Wendepunkt}

Das Thema: «Schweizer Geographie am Wendepunkt» weckt zwei Fragen:

1. Was heißt Schweizer Geographie?

2. Was heißt denn schon Wendepunkt?

Ich beginne mit der zweiten Frage, da sie uns ermöglicht, die Problemstellung deutlicher zu erfassen.

\section{Was heißt Wendepunkt in der Schweizer Geographie?}

Anknüpfend an die Einleitung zum Tagungsthema ist festzustellen: Die Geographie hat sich in den letzten 25 Jahren stark entwickelt, und dies in drei Richtungen. Einmal hat die Disziplin an praktischer Bedeutung gewonnen. Zum andern sind die Hochschulinstitute leistungsfähiger und spezialisierter geworden. Wesentlich ist ferner der Blick auf den Stellenmarkt. Während die traditionelle Tätigkeit in der Schule aus demographischen Gründen zurückgegangen ist, haben sich als Folge des verstärkten Umweltbewußtseins neue Tätigkeitsgebiete in der Praxis eröffnet.
Wie sehr die allgemeine Lage sich verändert hat, illustriert ein Blick auf die Entwicklung der Berufsfelder des Geographischen Institutes der Universität Bern im Zeitraum 1960 bis 1985 (Abb. 1).

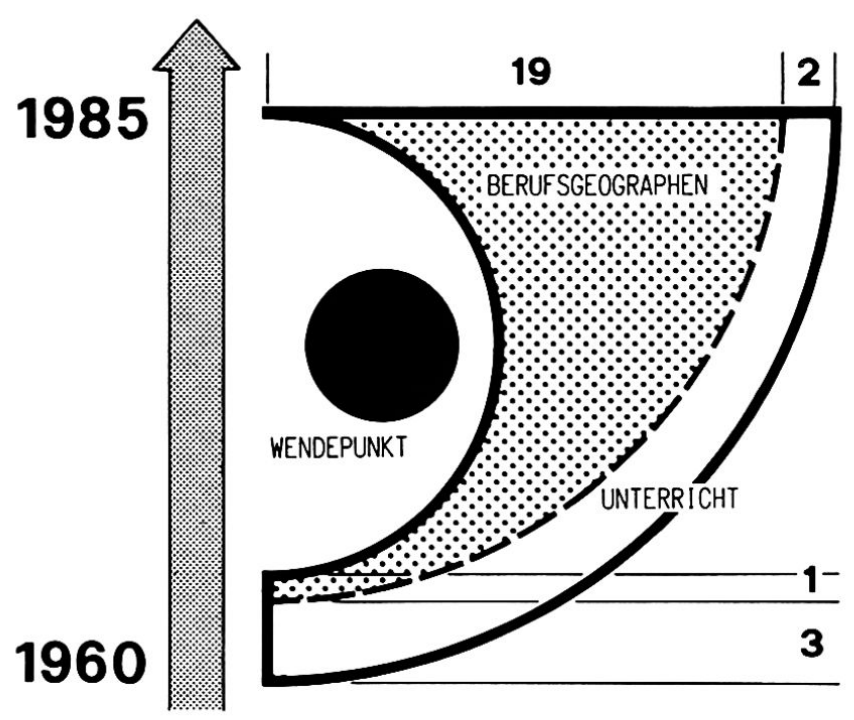

Abb. 1 Die Berufsfelder der Berner Geographen 1960 und 1985

Der «Wendepunkt» bedeutet:

1. Für die Geographen außerhalb der Universität: Der Stellenmarkt hat sich verändert.

2. Für die Geographen in der Universität: Der Fachinhalt, die Methoden und die Ausbildungsziele müssen überprüft werden.

3. Für die Geographen in der Schule: Das Schulfach ist in vermehrtem Maße entsprechend den Bedürfnissen unserer Gesellschaft weiter zu entwickeln.

Aus diesen drei Betrachtungen leite ich für die Universitätsinstitute folgende Fragen ab:

1. Nimmt die Universität diese Veränderungen wahr und berücksichtigt sie diesen Wandel in der permanenten Studienreform?

2. Wie kann das außerhalb der Universität entstehende Wissen der berufspraktischen Geographen in die Universität und damit in die Ausbildung eingebracht werden?

3. Wie kann die Verbindung Universität-Praxis-Schule gestärkt werden, so daß die Disziplin Geographie in der Öffentlichkeit an Konturen und damit an Einfluß gewinnt. 
点 Abb. 2: Übersicht der Lehrveranstaltungen im Grundstudium und im Hauptstudium

\begin{tabular}{|c|c|c|c|c|c|c|c|c|c|}
\hline \multirow{3}{*}{$\begin{array}{l}\text { Thematische } \\
\text { Kategorien }\end{array}$} & \multicolumn{5}{|c|}{ Formale Kategorien } & \multirow{2}{*}{\multicolumn{2}{|c|}{ Grundstudium }} & \multirow{2}{*}{\multicolumn{2}{|c|}{ Hauptstudium }} \\
\hline & \multicolumn{2}{|c|}{$\begin{array}{l}\text { Entdeckungs- } \\
\text { zusammenhang }\end{array}$} & \multirow{2}{*}{\begin{tabular}{|c} 
Begründungs- \\
zusammenhang
\end{tabular}} & \multicolumn{2}{|c|}{$\begin{array}{l}\text { Verwertungs- } \\
\text { zusammenhang }\end{array}$} & & & & \\
\hline & Theorie & Grundbegriffe & & $\begin{array}{l}\text { deskriptives } \\
\text { Wissen }\end{array}$ & $\begin{array}{l}\text { anwendungs- } \\
\text { bezogene } \\
\text { Ausbildung }\end{array}$ & $\begin{array}{l}\text { absolut } \\
\text { (Lektio- } \\
\text { nen) }\end{array}$ & $\%$ & $\begin{array}{l}\text { absolut } \\
\text { (Lektio- } \\
\text { nen) }\end{array}$ & $\%$ \\
\hline $\begin{array}{l}\text { Anthropo- } \\
\text { geographie }\end{array}$ & & & & & & 205 & 32 & 107 & 28 \\
\hline $\begin{array}{l}\text { Physische } \\
\text { Geographie }\end{array}$ & & & & & & 171.5 & 27 & 77,5 & 32 \\
\hline $\begin{array}{l}\text { Allgemein } \\
\text { integrative } \\
\text { Veranstaltungen }\end{array}$ & & & & & & 113 & 18 & 39 & 19 \\
\hline Methoden & & & & & & 143 & 23. & 83 & 21 \\
\hline $\begin{array}{ll} & \text { absolut } \\
\text { Grund- } & \text { (Lektionen) }\end{array}$ & 51 & 265 & 209 & 71 & 36,5 & 632,5 & - & - & - \\
\hline 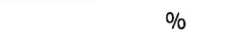 & 8 & 42 & 33 & 11 & 6 & - & 100 & - & - \\
\hline $\begin{array}{ll} & \text { absolut } \\
\text { Haupt- } & \text { (Lektionen) }\end{array}$ & 33 & 141 & 92 & 32 & 8,5 & - & - & 306,5 & - \\
\hline$\%$ & 4 & 39 & 42 & 14 & 11 & - & - & - & 100 \\
\hline
\end{tabular}

(Grundlage: Tab. A6 und A7 der Tagungsdokumentation) 
Abb. 3: Übersicht der Lehrveranstaltungen - Vergleich Deutschschweiz (D) - Romandie (R)

\begin{tabular}{|c|c|c|c|c|c|c|c|c|c|c|}
\hline \multirow{2}{*}{$\begin{array}{l}\text { Thematische } \\
\text { Kategorien }\end{array}$} & \multicolumn{5}{|c|}{$\begin{array}{l}\text { Formale Kategorien } \\
\mid \begin{array}{l}\text { Begründungs- } \\
\text { zusammenhang }\end{array}\end{array}$} & \multicolumn{3}{|c|}{ Grundstudium } & \multicolumn{2}{|c|}{ Hauptstudium } \\
\hline & Theorie & Grundbegriffe & Methoden & $\begin{array}{l}\text { deskriptives } \\
\text { Wissen }\end{array}$ & $\begin{array}{l}\text { anwendungs- } \\
\text { bezogene } \\
\text { Ausbildung }\end{array}$ & & $\begin{array}{l}\text { absolut } \\
\text { (Lektio- } \\
\text { nen) }\end{array}$ & $\%$ & $\begin{array}{l}\text { absolut } \\
\text { (Lektio- } \\
\text { nen) }\end{array}$ & $\%$ \\
\hline $\begin{array}{l}\text { Anthropo- } \\
\text { geographie }\end{array}$ & 22 & 22 & 22 & 22 & 22 & $\begin{array}{l}D \\
R\end{array}$ & $\begin{array}{r}98 \\
107\end{array}$ & $\begin{array}{l}30 \\
35\end{array}$ & $\begin{array}{l}91,5 \\
59\end{array}$ & $\begin{array}{l}24 \\
44\end{array}$ \\
\hline $\begin{array}{l}\text { Physische } \\
\text { Geographie }\end{array}$ & & & & & & $\begin{array}{l}\mathrm{D} \\
\mathrm{R}\end{array}$ & $\begin{array}{l}94 \\
77,5\end{array}$ & $\begin{array}{l}29 \\
25\end{array}$ & $\begin{array}{r}145 \\
23\end{array}$ & $\begin{array}{l}37 \\
16\end{array}$ \\
\hline $\begin{array}{l}\text { Allgemein } \\
\text { integrative } \\
\text { Veranstaltungen }\end{array}$ & & & & & & $\begin{array}{l}D \\
R\end{array}$ & $\begin{array}{l}74 \\
39\end{array}$ & $\begin{array}{l}23 \\
13\end{array}$ & $\begin{array}{l}60 \\
41\end{array}$ & $\begin{array}{l}15 \\
30\end{array}$ \\
\hline Methoden & & & & & & $\begin{array}{l}\mathrm{D} \\
\mathrm{R}\end{array}$ & $\begin{array}{l}60 \\
83\end{array}$ & $\begin{array}{l}18 \\
27\end{array}$ & $\begin{array}{l}93 \\
14\end{array}$ & $\begin{array}{l}24 \\
10\end{array}$ \\
\hline $\begin{array}{ll}\text { absolut } \\
\text { Grund- (Lektionen) }\end{array}$ & $\begin{array}{l}18 \\
33\end{array}$ & $\begin{array}{l}124 \\
141\end{array}$ & $\begin{array}{r}117 \\
92\end{array}$ & $\begin{array}{l}39 \\
32\end{array}$ & $\begin{array}{l}28 \\
8,5\end{array}$ & $\begin{array}{l}D \\
R\end{array}$ & $\begin{array}{l}326 \\
304,5\end{array}$ & - & - & - \\
\hline studium & $\begin{array}{r}5 \\
11\end{array}$ & $\begin{array}{l}38 \\
46\end{array}$ & $\begin{array}{l}36 \\
30\end{array}$ & $\begin{array}{l}12 \\
10\end{array}$ & $\begin{array}{l}9 \\
3\end{array}$ & $\begin{array}{l}D \\
R\end{array}$ & - & $\begin{array}{l}100 \\
100\end{array}$ & $\begin{array}{l}- \\
-\end{array}$ & - \\
\hline $\begin{array}{ll}\text { absolut } \\
\text { Haupt- (Lektionen) }\end{array}$ & $\begin{array}{l}12 \\
10\end{array}$ & $\begin{array}{r}151 \\
53\end{array}$ & $\begin{array}{r}138 \\
32 \\
\end{array}$ & $\begin{array}{l}45 \\
29\end{array}$ & $\begin{array}{l}43,5 \\
13\end{array}$ & $\begin{array}{l}D \\
R\end{array}$ & - & - & $\begin{array}{l}389,5 \\
137\end{array}$ & - \\
\hline studium & $\begin{array}{l}3 \\
7\end{array}$ & $\begin{array}{l}39 \\
39\end{array}$ & $\begin{array}{l}35 \\
23\end{array}$ & $\begin{array}{l}12 \\
21\end{array}$ & $\begin{array}{l}11 \\
10\end{array}$ & $\begin{array}{l}D \\
R\end{array}$ & - & $\begin{array}{l}- \\
-\end{array}$ & $\begin{array}{l}- \\
-\end{array}$ & $\begin{array}{l}100 \\
100\end{array}$ \\
\hline
\end{tabular}




\section{Was heißt Schweizer Geographie?}

Um auf diese Frage antworten zu können, sind im Rahmen derVorbereitung derTagung umfangreiche Befragungen durchgeführt worden.

a) Die Gruppe um Martin Schuler in Lausanne untersuchte das "Selbstbild der Schweizer Geographen 1985». Die Ergebnisse bilden Teil 2 der Tagungsdokumentation.

b) Die Gruppe um Dr. BennoWerlen in Zürich verglich die heutigen Ausbildungsprogramme der Universitäten. Die Resultate sind unter dem Titel «Das implizite Leitbild der Geographie an den Geographischen Instituten der Schweizer Hochschulen» im Teil 3a der Tagungsdokumentation enthalten.

c) Die Gruppe der SGAG um Dr. Charles Hussy und Ueli Roth überprüfte die Beurteilung der Geographen durch die Brille der Arbeitgeber. Das Fazit dieser Umfrage hat Charles Hussy vorn im Kapitel 2.3 vorgelegt.

Hier steht nun zur Diskussion die Frage:

Wie präsentiert sich die Schweizer Geographie im Rahmen der Ausbildungsprogramme? Die Grunddaten zu dieser Fragestellung sind in Teil 3a der Tagungsdokumentation niedergelegt.

\section{II.1 Das Grundstudium in Geographie an den Schweizer Hochschulen}

Abb. 2 unterscheidet «Formale» Kategorien und «Thematische» Kategorien. Die «Formalen» Kategorien nehmen im Sinne POPPERS Bezug auf dessen Gliederung jeglicher wissenschaftlicher Tätigkeit in die Bereiche «Entdeckungszusammenhang», «Begründungszusammenhang» und «Verwertungszusammenhang». Die «Thematischen» Kategorien entsprechen der traditionellen Gliederung der Fachdisziplin. Über die Gliederungsweise des vorgelegten Rasters läßt sich streiten. Es hat aber kein Institut einen besseren Vorschlag eingebracht.

Es geht hier darum, eine Brille, d. h. eine Betrachtungsmöglichkeit für die unterschiedlichen Ausbildungsstrukturen zu erhalten. Die Angaben sollen daher vor allem zum Nachdenken anregen. In gekürzter Form läßt sich für den Aufwand in der Lehre feststellen:

1. die Anteile «Theorie» und «Anwendungsbezogene Ausbildung» sind schwach ausgebildet.

2. Der Anteil der Grundbegriffs-Veranstaltungen ist hoch.

3. Einem schwachen Theorieanteil steht ein stark dotierter Methodenanteil gegenüber.

4. Die Detailangaben (Tab. A6 ff der Tagungsdokumentation) zeigen geringe Anteile für integrative Veranstaltungen und Ökologie.

\section{II.2 Das Hauptstudium}

Abb. 2 belegt, daß sich das Hauptstudium in den Proportionen der verschiedenen Kategorien nur unwesentlich vom Grundstudium unterscheidet. $\mathrm{Zu}$ sammenfassend ergibt sich:

1. Abb. 2 zeigt die Breite des Faches sowohl im Grund- wie im Hauptstudium.

2. Es wird nicht ersichtlich, wie im Hauptstudium die Akzente gesetzt werden, da ja je nach Universität aus dem gesamten hier aufgenommenen Angebot nur ein Teil davon für die Absolventen obligatorisch ist.

3. Angesichts der Breite des Angebots stellt sich die Frage, welche Akzente berufsbezogen von besonderer Bedeutung sind.

4. Es ist im Einzelnen zu prüfen, welche Bereiche zu schwach dotiert sind oder sogar fehlen und ob diese fehlenden Bereiche in das Hauptstudium oder in ein zu errichtendes Nachdiplomstudium einzubauen wären.

\section{II.3 Der Vergleich des Lehrangebotes in der Deutschschweiz und in der Romandie}

Beim Vergleich des Grundstudiums in Geographie zwischen Deutschschweiz und Romandie zeigen sich folgende Beobachtungen (Abb. 3):

1. Die Grundtendenz entspricht dem gesamtschweizerischen Verteilungsbild (vgl. Abb. 2).

2. Der Anteil der integrativen Veranstaltungen liegt in derWestschweiz tiefer als in der Deutschschweiz.

3. Im Anteil der Methoden liegen die Verhältnisse umgekehrt.

4. In der Westschweiz sind gegenüber der Deutschschweiz der Theorieanteil höher, umgekehrt sind die Anteile im Bereich Anwendungsbezogener Ausbildung.

Im Hauptstudium weichen die Romandie und die Deutschschweiz stärker voneinander ab (Abb. 3):

1. Die Unterschiede liegen weniger in den «Formalen» Kategorien als vielmehr in den «Thematischen» Kategorien.

2. Die starken Unterschiede in den «Thematischen» Kategorien gehen vermutlich darauf zurück, $\mathrm{da} ß$ in der Westschweiz nicht alle Institute in den naturwissenschaftlichen Fakultäten eingeordnet sind. Hier spiegelt sich offenbar die Vielfalt der schweizerischen Universitätsstrukturen wider.

3. Der Hauptunterschied liegt in der Stundendotation, indem die vier westschweizerischen Institute insgesamt nur 137 Lektionen anbieten, wogegen die vier deutschsprachigen Institute über ein Angebot von 390 Lektionen verfügen. 
4. In der Westschweiz hat man offenbar aus dieser Einsicht die Konsequenz gezogen und einen troisième cycle eingerichtet, der periodisch die fehlenden Teile bringt.

Abschließend eine allgemeine Feststellung: Die Auslegeordnung der Programme hat gezeigt, da $\beta$ eine große Breite vorhanden ist. Hier liegt eine Chance, hier liegen auch Gefahren. Es wäre wünschenswert zu wissen, ob auch unsere beruflichen Konkurrenzfächer derartige Breiten aufweisen oder ob dort bewußt auf engen Fachausschnitten gearbeitet wird.

Die folgenden Beiträge der Herren Dr. Werlen und Dr. Boesch bringen nun Folgerungen aus der eben dargelegten Studienstruktur.

\subsection{B) B. Werlen: Überlegungen zu einem Leitbildentwurf für die Ausbildung von Diplom-Geographen}

Die von Prof. Aerni soeben kommentierte Radiographie der aktuellen Ausbildungssituation an den Schweizer Hochschulen zeigt, daß sich angehende Geographinnen und Geographen heute mit einem thematisch äußerst umfassenden und heterogenen Ausbildungsprogramm konfrontiert sehen. Daraus ergeben sich verschiedene potentielle Konsequenzen, die ich als Mitglied der Zürcher Arbeitsgruppe «Wissenschaft und Methoden» anhand von vierThesen zusammenfassen möchte (vgl. BARTELS, 1973, 24f.; WERLEN, 1986). In der darauf aufbauenden Argumentation gehe ich auch auf Fragen ein, die von der SGK, SGgG und SGAG an unsere Arbeitsgruppe herangetragen wurden.

These 1: Ein thematisch zu umfassendes und heterogenes Ausbildungsprogramm gefährdet den Fortbestand der Geographie als wissenschaftliche Disziplin.

Die große Pluralität der Ausbildungsinhalte hat in der jüngsten Zeit zu einer immer stärkeren Bezugnahme auf die Nachbardisziplinen geführt. Beginnen schließlich die einzelnen Subdisziplinen der Geographie mit ihren Nachbardisziplinen mehr Gemeinsamkeiten aufzuzweisen als mit den übrigen Fachbereichen der Geographie, dann gibt es schließlich keine guten Gründe mehr, die institutionelle Einheit als wissenschaftliche Disziplin aufrechtzuerhalten.

Auch in der Einleitung zur Tagungsdokumentation wird der Umstand beklagt, daß die Öffentlichkeit das Zuständigkeitsmaß der Geographen zur Lösung von sogenannten «Raumproblemen» als niedrig einstuft. Darauf nimmt

These 2 Bezug. Sie lautet: Ein thematisch zu umfassendes und heterogenes Ausbildungsprogramm verunmöglicht es, ein einheitliches Öffentlichkeitsimage sicherzustellen.

Ist die Vielfalt der vermittelten Ausbildungsinhalte so groß, daß selbst Geographen Mühe haben, den Zuständigkeitsbereich der Geographie als einheitliche Disziplin eindeutig zu definieren, dann ist es auch verständlich, da $\beta$ die Erwartungen der Öffentlichkeit an unsere Disziplin diffus bleiben. Bei genauerer Betrachtung könnte es sich aber auch erweisen, daß die Geographie gar keine Öffentlichkeit besitzt und diese erst geschaffen werden sollte. Einen Grund, warum möglicherweise ein mangelndes Öffentlichkeitsinteresse für Leistungen der Geographie besteht, möchte ich im Sinne einerVermutung in der nächsten These bzw. Hypothese formulieren:

These 3: Ein thematisch zu umfassendes und zu heterogenes Ausbildungsprogramm verhindert die wissenschaftliche und praktische Konkurrenzfähigkeit geographischer Forschungsarbeit.

Denn bei zu großerThemenvielfalt leidet die ausreichende Vertiefung der Lern- und Lehrinhalte, so daß die Forschungskompetenz tendenziell auf der deskriptiven Stufe stehenbleibt. Anhand dieser Voraussetzung können Geographinnen und Geographen nur unter erschwerten Bedingungen sowohl im wissenschaftlichen als auch in anwendungsbezogenen Berufsbereichen konkurrenzfähig werden.

Geht man davon aus, daß das Ziel wissenschaftlicher Tätigkeiten in der Bildung gültiger Theorien liegt, aus welchen zur Lösung praktischer Probleme plausible und erfolgreiche Vorgehensweisen abgeleitet werden können, dann wird ein weiterer Mangel im aktuellen Leitbild der Geographie offensichtlich. Dazu die vierte und letzte These:

These 4: Wenn im Rahmen einer wissenschaftlichen Disziplin längerfristig eine sinnvolle Forschung geleistet werden will, die auch einen erfolgreichen Praxisbezug erlaubt, dann sollten die Fachvertreter explizit und systematisch auf Theoriebildung hinarbeiten. Die bestehenden Fachtheorien sollen zu den Orientierungsleitlinien der Forschungsvorhaben sowie der Wissenschaftsanwendung gemacht werden, so daß sie in der Praxis immer wieder überprüft und revidiert werden können. 
Ist diese These nicht unzutreffend und möchte man die entsprechenden Forderungen verwirklichen, dann sollten in Zukunft unsere Ausbildungsprogramme einen Schwerpunkt im Bereich der Fachtheorien aufweisen. Zudem wäre die Ausbildung in empirischer Forschungsmethodik theoriebezogen zu vermitteln, so daß die Hinführung zum Praxisbezug erleichtert wird.

In diesem Zusammenhang sei eine Zwischenbemerkung zu einem Umfrageergebnis der Arbeitsgruppe «Selbstbild der Schweizer Geographen» erlaubt. Es wurde festgestellt, daß die Absolventen der Westschweizer Universitäten den Gewinn theoretischer Kenntnisse für ihre Berufstätigkeit niedrig einschätzen, obwohl hier die Theorie weniger stark vernachlässigt wird als anderswo, und daß die Zürcher Geographen die erlernten Techniken und Methoden schlecht benoten, obwohl hier ihr Ausbildungsschwergewicht liegt. Dieses Resultat sollte aber nicht zu dem Schluß verleiten, daß ein gutes universitäres Angebot in einem Bereich eine schlechte Benotung der Nützlichkeit für den Berufsalltag provoziert. Es wäre vielmehr auch die Interpretationsmöglichkeit ins Auge zu fassen, daß die Ausbildung negativ bewertet wird, weil sie nicht integriert vermittelt wurde. Damit meine ich, daß die Theorie vielleicht ohne ausreichenden Bezug zur empirischen Forschungsmethodik und zur praktischen Umsetzung und die Ausbildung in Methoden und Techniken ohne ausreichenden Theorie- und Praxisbezug bleibt.

Nun aber wieder zurück zum Problem des Theoriedefizits. Wenn ein stärkerer Theoriebezug in der Ausbildung verwirklicht wird, sollte dies nicht zu einem beliebigen «Wildwuchs» handgestrickter Theoriekonstrukte führen. Die zu fördernden Theorien sollten bestimmten formalen Mindestkriterien genügen. Unter Bezugnahme auf einen Gedankengang von Dietrich Bartels (vgl. BARTELS, 1970, 456f.) und einer Weiterentwicklung seiner Anregung (vgl. WERLEN, 1986) sind die folgenden Mindest- bzw. Relevanzkriterien für geographische Fachtheorien zu nennen:

Sie sollten erstens praktische Relevanz aufweisen,

d. h. auch, daß sie dem Diplom-Geographen für seine spätere Berufstätigkeit dienlich sein sollen.

Sie sollten zweitens didaktische Relevanz aufweisen,

d. h. daß das anhand von ihnen gewonnene Wissen dem Schüler für seine Lebenspraxis dienlich sein soll, was nicht zuletzt auch zur Image-Verbesserung beitragen könnte.

Sie sollten drittens interdisziplinäre Relevanz aufweisen,

d.h. a) daß geographische Forschung in einer Sprachregelung betrieben werden sollte, die mit den Vertretern anderer Disziplinen eine unverzerrte Kommunikation ermöglicht, und b) da $\beta$ diese Theorien einen expliziten Bezug zu den Theorien der Sozial- und Naturwissenschaften aufweisen sollten, indem sie diese in räumlicher Hinsicht erweitern. Da sich das Image einer Disziplin in der Regel an der Bedeutung ihrer Ergebnisse für die Nachbardisziplinen bemisst, dürfte dies eine nicht zu unterschätzende Forderung sein. Dazu kommt, daß Interdisziplinarität eine gemeinsame Sprache voraussetzt.

Sie sollten viertens wissenschaftstheoretische Relevanz aufweisen (vgl. WERLEN, 1986),

d. h. daß die geographischen Theorieansätze und die auf ihnen aufbauenden Forschungspraktiken mit dem erkenntnistheoretischen und methodologischen Wissen in Einklang stehen müssen. Mit der Erfüllung dieses vierten Kriteriums sollte insbesondere die Verbesserung der Qualität der Forschungsergebnisse erreicht werden, was schließlich zur Erhöhung der Relevanz geographischer Forschung in praktischer, didaktischer und interdisziplinärer Hinsicht beitragen könnte.

Die Frage ist nun, wo man mit den verändernden Maßnahmen einsetzen sollte. Die Maßnahmen, die für die Sicherung des Fortbestandes der Geographie als wissenschaftlicher Disziplin, zur Schaffung eines einheitlichen Öffentlichkeitsimages und zur Hebung der Konkurrenzfähigkeit geographischer Forschungsarbeiten ergriffen werden, sollten zuerst in der Neugestaltung des.Grundstudiums ihren Niederschlag finden. Dafür kann man mehrere Gründe anführen. Zunächst weil hier die Einübung in die geographische Betrachtungsweise stattfindet und somit jede Veränderung am sinnvollsten von dieser Ebene ausgehen muß. Zweitens kann mit der Vereinheitlichung des Grundstudiums die notwendige Voraussetzung für einen Universitätswechsel nach der ersten Studienhälfte geschaffen werden. Sobald diese Bedingung erfüllt ist, wird es möglich, an den einzelnen Instituten sinnvolle Spezialisierungen des Fachstudiums und der Forschungsbereiche anzustreben. Gleichzeitig sollte unter den Instituten eine Abstimmung der Forschungsbereiche erreicht werden. Auf diese Weise könnte dann der "Zersplitterung der Kräfte» vorgebeugt werden, ohne daß sich Lehre und Forschung zu entfremden brauchen.

Auf der Ebene des Grundstudiums wären konkret folgende Maßnahmen vorzusehen:

1. Vereinheitlichung der Einführungsveranstaltungen in Anthropogeographie, Physischer Geographie und der korrelativen Forschungsmethodik.

2. Vereinheitlichung und Einschränkung des thematischen Ausbildungsspektrums. 
3. Standardisierung der Basiskurse bzw. Unterseminarien in den als zentral betrachteten Ausbildungsbereichen.

Welche Themenbereiche die Ausbildung allerdings umfassen soll, müßte von einer erst zu schaffenden Kommission entschieden werden, in der die verschiedenen Fachgesellschaften, die Professoren und der Mittelbau ebenso vertreten sein sollten wie die studentischen Fachvereine. Der Entscheidungspro$z e ß$ sollte offen und kooperativ gestaltet werden und die zuvor formulierten Mindestkriterien nicht unbeachtet lassen.

Es ist klar, daß unter den schweizerischen Bedingungen, d.h. im Rahmen der föderalistischen Hochschulstruktur und der Tatsache, daß sich die Geographischen Institute an drei verschiedenen Fakultäten befinden, dieses Vorhaben nur schwierig zu verwirklichen ist. Will man aber die aktuelle forschungspolitische Situation sowie die Relevanz der Forschungsergebnisse der Geographie verbessern, dürften die Vereinheitlichung der Ausbildung im Grundstudium sowie ein verstärkter Theorie- und Praxisbezug eine nicht zu unterschätzende Bedeutung aufweisen. Die vorgetragenen Vorschläge sind primär nur als hypothetische Konsequenzen zu begreifen, die sich ergeben, wenn man die gesetzten Ziele: d. h. die Verbesserung der Identität des Geographen, die Verbesserung der Situation auf forschungspolitischer Ebene, die Hebung des Öffentlichkeitsimages und die Verhinderung der Aufsplitterung der Kräfte erreichen will. Die Frage ist natürlich, ob man diese Ziele erreichen will und ob nicht alternative Mittel der Zielerreichung bestehen. Antworten auf diese Fragen und Stellungnahmen zu den hier unterbreiteten Überlegungen zu formulieren, sollte der Gegenstand der Diskussion in der Gruppe «Geographie an der Hochschule» und der Plenumsdiskussion von morgen sein.

\section{Literatur}

BARTELS, D. (1970): Zwischen Theorie und Metatheorie. In: Geographische Rundschau, 22, S. 451-457.

BARTELS, D. (1973): Between theory and metatheory. In: CHORLEY, R. (ed.): Directions in Geography. London, S. 25-37.

WERLEN, B. (1986): Zwischen Metatheorie, Fachtheorie und Alltagswelt. Eine Auseinandersetzung mit Bartels' Stufenmodell anwachsender Rationalität wissenschaftlichen Handelns. In: BAHRENBERG, G./DEITERS, J./GAEBE, W./FISCHER, M./HARD, G./LOEFFLER, G. (Hrsg.): Geographie des Menschen. Gedenkschrift für Dietrich Bartels, Bremen, 1986 (im Druck).
2.4 C) Martin Boesch: Schweizer Geographie am Wendepunkt: Überlegungen zu einer Normativen Metatheorie*

\section{Vorbemerkungen}

Schweizer Geographie am Wendepunkt? - Ob die Schweizer Geographie tatsächlich an einem Wendepunkt angelangt ist und in Zukunft neue Wege beschreiten wird oder ob es so weitergeht wie bisher, ist wohl eine offene Frage. Es liegt an uns, auf diese Frage eine positive Antwort zu geben - durch unsere Arbeit als Geographen. Aus der Reflexion über den Sinn unseres Tuns können wir dafür neue Impulse schöpfen. Mein Referat will dazu einige Gedanken beisteuern. Bei meinen Überlegungen lasse ich mich leiten von der Erwartungshaltung, die heute bereits mehrmals deutlich zum Ausdruck gekommen ist: von der Erwartung nämlich, da $\beta$ unsere Disziplin vermehrt zu Problemlösungen ${ }^{1}$ beitragen soll.

Der im Titel angedeutete Bezug zur «New Age»-Bewegung (F.CAPRA; M. FERGUSON) ist unübersehbar. Er signalisiert unsere heutige Siutation: ein Ende der bisherigen Selbstverständlichkeit, ein Suchen nach neuen Zielen und Wegen, ein Bedürfnis nach Standortbestimmung und Neuorientierung. ${ }^{2}$ Dabei nimmt die Überwindung des «linearen Denkens» eine zentrale Rolle ein - und die Thesen von N. POSTMAN gewinnen eine unerwartete Bedeutung. Ihm geht es ja um die Rolle des Fernsehens in unserer Gesellschaft, insbesondere auch um dessen Einfluß auf die Entwicklung von Kindern und Jugendlichen. Dabei manifestiert sich ein Gegensatz zwischen bildhaftem («unmittelbarem») Sehen und Begreifen einerseits und analytischem Denken andererseits. Neurobiologische und entwicklungsphysiologische Befunde unterstützen diese Thesen. $\mathrm{Ob}$ man - wie POSTMAN - das Fernsehen als Gefahr für die Entwicklung der traditionellen analytischen Intelligenz begreifen will, mag hier offen bleiben. Für uns ist die Umkehrung der Argumentation bedeutungsvoll: ist nicht die stärkere Vernetzung beider Denkmodi, des analytischen und des ganzheitlichen, gerade eine Chance? Die Konturen eines neuen Wissenschaftsbildes zeichnen sich ab; bei vielen Projekten ganz verschiedener Fachbereiche wer-

\footnotetext{
* Überarbeitete Fassung des Referates vom 2. Mai 1986 in Bern
} 
den heute solche «Grenzüberschreitungen» sichtbar. Und aus der Berührung verschiedener Denkansätze und Partialtheorien ergeben sich überraschende neue Erkenntnisse, gerade auch im Berührungsfeld analytischer und makroskopischer Ansätze. Damit gewinnt der Begriff der «Komplementarität» (W. J. COFFEY 1981/30) auch für die Geographie erhöhte Aktualität. Könnte die Geographie dabei nicht eine aktive Rolle spielen - nicht im Sinne einer eigennützigen und letztlich schädlichen Kirchturmpolitik, sondern zur allgemeinen Förderung solcher Ansätze?

\section{Zum Wissenschaftstheoretischen Pluralismus}

Unter «Wissenschaft» bzw. "wissenschaftlich» Arbeiten verstehe ich alle Tätigkeiten, die «Wissen» nicht nur neu gewinnen (generieren), sondern auch «Wissen» umformen und vermitteln (transformieren und transferieren). Dabei meint «Wissen» nicht einfach beliebige Kenntnisse oder Aussagen, sondern allgemeine, systematisch geordnete Sätze über die «Welt», sei sie nun real oder imaginär. Demgegenüber haben Basissätze den Stellenwert von propädeutischem Wissen. Mit andernWorten: auch praxisbezogene und didaktische Leistungen können (je nachdem) das Prädikat "wissenschaftlich" beanspruchen; Diskussionen über neue Ziele und Wege der «Wissenschaft» gehen auch den Praktiker und den Lehrer etwas an. Das breitgefächerte Auditorium an dieser Tagung bestätigt dies eindrücklich. Wissenschaftstheorie schließlich macht Aussagen über die Qualität solcher «allgemeiner Sätze», und wie man sie gewinnt. ${ }^{3}$

Die Vorstellung von einer monolithischen Wissenschaftstheorie, im Sinne des Kritischen Rationalismus, muß heute als überholt bezeichnet werden. Es gibt sie nicht, die umfassende Einheitsmethodologie; sie hat sich verflüchtigt unter der berechtigten Kritik des Reduktionismus. Die Divergenz der Auffassungen ist vielschichtig; der wissenschaftstheoretische Pluralismus ist (heute) eine Realität. Vgl. dazu Abb. 1. Wir unterscheiden zwei Hauptsegmente, nämlich analytische und nicht-analytische Ansätze. Quer dazu verlaufen die vier Stufen wissenschaftlichen Arbeitens: die deskriptive, die explikative, die normative und die operative Stufe. ${ }^{4}$ Wir können daraus den Schluß ziehen, daß die Auffassungen von "Wissenschaft», von "wissenschaftlichem» Arbeiten und «Wissenschaftlichkeit» sehr heterogen sind. Ferner gilt, da $B$ dieser Pluralismus mehr oder weniger quer durch alle Disziplinen hindurch verläuft; mit Sicherheit trifft das für die Geographie zu. ${ }^{5}$
Und daraus ergibt sich zwingend eine erste Forderung an eine zukunftsorientierte Hochschulausbildung (und auch an die Forschungspraxis): wir sollten in bezug auf diese breite und dynamische wissenschaftstheoretische Diskussion mehr Transparenz herstellen, wir sollten über wissenschaftstheoretische Ansätze diskutieren können, auch Absolventen unserer Institute sollten eine bewußte Standortbestimmung vornehmen können, sie sollten mit den Richtungen und Schulen, die heute vertreten werden, etwas anfangen können. Es geht also primär um wissenschaftstheoretische Reflexion und Mitsprachekompetenz. Ich möchte allerdings noch einen Schritt weitergehen: es genügt nicht, andere Ansätze wohlwollend zu ignorieren, sondern wir sollten einen «Kooperativen Pluralismus» erreichen, aktive Versuche grenzüberschreitender Kontakte (im methodologischen Sinne) unternehmen. Auf diesem Feld besteht zweifellos noch ein gewisses Defizit, das behoben werden sollte.

\section{Zum Normendefizit in der Geographie}

Gelegentlich mißversteht man unter «Anwendung» einer Disziplin die platte Verwendung ihres Basiswissens und ihrer Methodik in einem beliebigen Kontext. Dies ist eine rein instrumentelle Auffassung: die Wissenschaft als Wasserträger der Verwertungsinteressen. Anwendung im eigentlichen Sinne bedeutet dagegen mehr: Stellungnehmen zu aktuellen gesellschaftlichen Problemen und Lösungsansätze erarbeiten, unter expliziter (wo nötig: kritischer) Bezugnahme auf Ziele und Normen, auf die Sinnfrage. Eine Disziplin verurteilt sich selbst zur Bedeutungslosigkeit, wenn sie nicht in sich den $\mathrm{Zu}$ sammenhang von

«Zielsetzung $\rightarrow$ Analyse $\rightarrow$ Therapie $\rightarrow$ Handlung» ermöglicht. Es scheint mir deshalb sinnvoll zu sein, kurz auf die Bedeutung von Theorien und Normen im Kontext wissenschaftlichen Arbeitens einzugehen. Daraus werden die entsprechenden Schlußfolgerungen, die ich ziehen werde, verständlich. Ausgangspunkt sind die vier Stufen wissenschaftlichen Arbeitens (vgl. Abb. 1 und 2).

Auf der ersten Stufe handelt es sich um ein deskriptives Wissenschaftsverständnis. Fakten und Logik (Formalwissenschaften) stehen unverbunden nebeneinander. Durch ihre Verknüpfung werden Theorien generiert; wir stehen auf der explikativen Stufe. Dies ist das Bild einer wertfreien Wissenschaft, etwa im Kritischen Rationalismus, im Szientismus.

Dieses Bild gilt heute zu recht als unvollständig: Normen und Werterhaltungen (gesellschaftliche 
bzw. individuell wirksame Werte) spielen eine Rolle, die nicht ignoriert werden darf. Durch den Einbezug von Wertungen und Normen ergeben sich zwei Effekte: einmal ein konstruktivistischer Effekt, d. h. Fakten werden entsprechend bestimmter

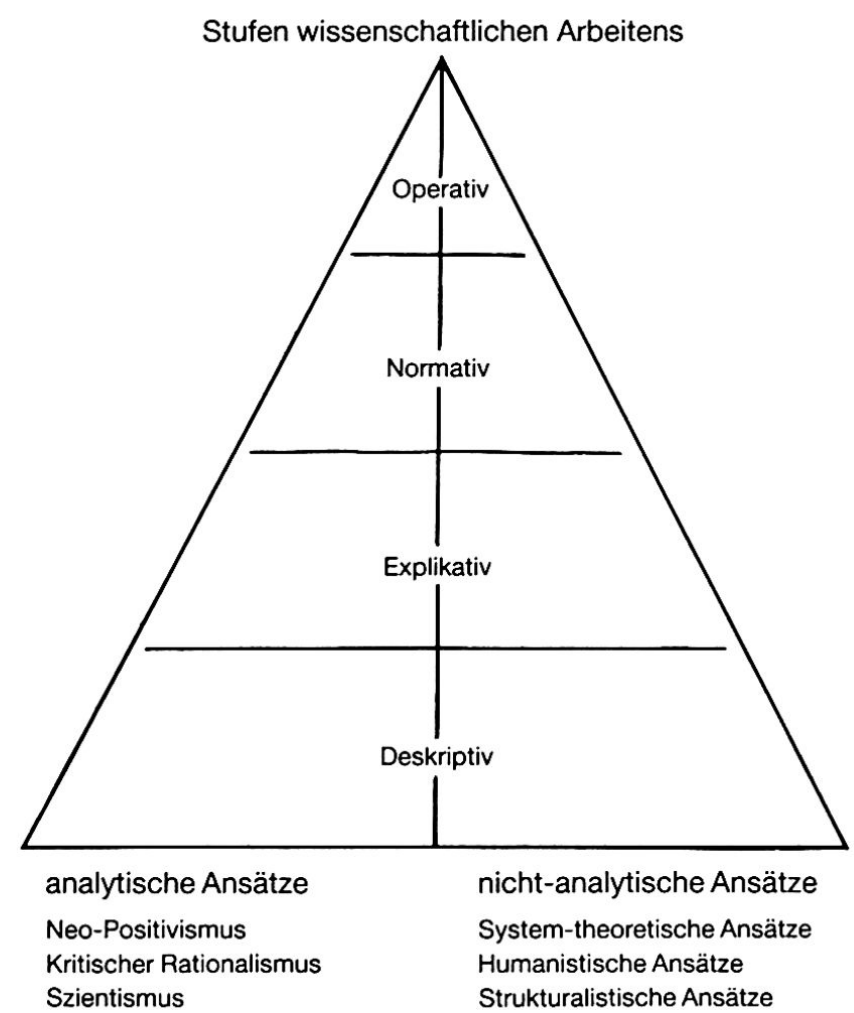

Abb. 1 Der wissenschaftstheoretische Pluralismus ist eine Realität. Es gibt keine Einheitsmethodologie.

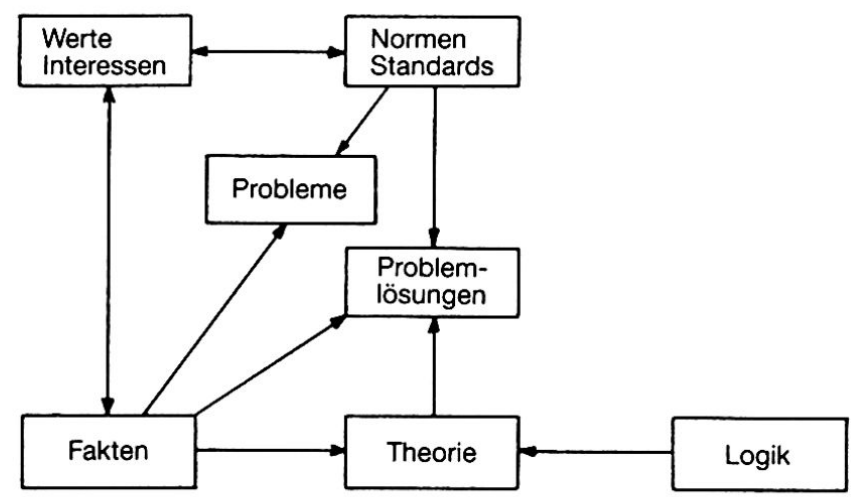

Abb. 2 Problemlösungen entstehen durch die Verknüpfung von Fakten, Theorie und Normen.
Werthaltungen ausgewählt bzw. ausgeblendet, die «Realität» wird erst durch das Subjekt konstruiert. Umgekehrt wirkt diese «Realität» aber auch zurück auf die Normen; sie sind einem Wandel unterworfen. Zum zweiten entsteht durch die Konfrontation von Fakten und Werten ein Problembewußtsein; Probleme werden also verstanden als Diskrepanz zwischen Soll- und Istzustand. Dies ist die normative Stufe.

Wenn wir unter dem Leitmotiv «Problemlösung» handeln wollen, müssen wir noch einen letzten Schritt tun: wir müssen Theorien, Sachverhalte (Fakten) und Normen miteinander in Beziehung bringen und kommen so zu Problemlösungen. J. MITTELSTRASS hat einmal die Handlungswissenschaften als «Moral Science» bezeichnet, um den inhärenten Zusammenhang von Fakten, Werten und Handlung zu betonen. Dies ist die operative Stufe. Wir sehen daraus: es gibt keine theoriefreie und keine wertfreie Problemlösung. In diesem Sinne sind die später formulierten Forderungen nach verstärktem Theoriebezug und dem expliziten Einbezug von Normen in unsere Ausbildung nicht Selbstzweck, sondern schlichte Konsequenz; wer Problemlösungen anstrebt, kommt nicht darum herum. ${ }^{6}$

Nun sind allerdings die Konturen einer aktiven oder «stellungnehmenden» Geographie nur schwach zu erkennen. ${ }^{7}$ Unserer Geographie mangelt diese Fortentwicklung zur normativen und operativen Stufe weitgehend; wir stellen ein sehr ausgeprägtes Normendefizit fest, und zwar ein dreifaches:

- Auf der untersten (oder wohl besser: innersten) Ebene fehlt es an Transparenz der «Wertbasis». Damit ist der persönliche Standort jedes einzelnen gemeint. Der «Wissenschaftler» läßt sich nicht trennen vom «Bürger», beide sind in einer Person stets verknüpft, all sein Tun und Lassen ist wert- oder interessengeleitet. Diese Erkenntnis sollte auch in der Geographie stärker betont werden, und es sollte daraus die Konsequenz gezogen werden: daß wir hier Transparenz schaffen. Das hat nichts zu tun mit einerVereinheitlichung oder gar Gleichschaltung, sondern mit dem Offenlegen dieser Wertbasis.

- Zweitens stellen wir eine mangelnde «Werterhellung» fest, d. h. es fehlen uns genügende Kenntnisse über Zusammenhänge zwischen Werten, Handlungen und der «Realität». Was passiert eigentlich in diesem Prozeßablauf, welches sind die Interessen, Werthaltungen und damit verbundenes Problembewußtsein, die zu konkreten Handlungen und Ereignissen führen? Und wie verändern sie die Realität? Umgekehrt: welches sind die Entscheidungskriterien, wenn es um konkrete Maßnahmen geht? Wenn wir Problemlösungen anstreben, müssen wir Kenntnisse haben über die 
Zusammenhänge zwischen sich konkurrierenden Oberzielen und wie denn aus solchen Ziel-MittelHierarchien schließlich konkretes Handeln abgeleitet werden kann, welche Maßnahmen im Sinne der Zielsetzung auch tatsächlich greifen. Wir brauchen also diese Werterhellung (insbesondere auch als Basis einer Theorie raumwirksamer Maßnahmen), um vernüftig handeln zu können.

- Schließlich konstatieren wir wohl ein ungenügendes "Wertengagement»: Erkenntnisse bereitstellen genügt allein meist nicht; isoliertes Wissen bringt selten etwas in Bewegung. Man muß abstraktes Wissen umsetzen in Problemlösungen und solche Vorschläge auch vertreten, zum Beispiel mit Öffentlichkeitsarbeit, in der Politik, selbstverständlich in den Schulen und in der Praxis. Mitwirkung in Entscheidungsprozessen ist die direkteste Form angewandter Wissenschaft. Nur indem wir hier mehr tun als bisher, werden wir als Disziplin auch ernstgenommen.

Das Normendefizit in der Geographie, die mangelnde Aussagekraft ihrer Ergebnisse, ist ein ganz zentraler Punkt ihrer Schwäche. Die Geographie hat ihre politische Unschuld noch weithin bewahrt; der Preis dafür ist ihre Belanglosigkeit.

\section{Normative Metatheorie}

Sollen sie nicht steril sein, müssen aus der Standortbestimmung, aus der Kritik konstruktive Vorschläge erwachsen. Das ist die Frage nach dem (neuen?) Leitbild der Geographie. Auch bei dieser Diskussion (soweit sie überhaupt geführt wird), muß ein Normendefizit konstatiert werden: implizite Antworten auf die gestellte Frage durch den «Tatbeweis» der täglichen Arbeit sind oft unreflektiert und vernachlässigen einen klaren Bezug auf die gültigen Relevanzkriterien (d. h. Normen) wissenschaftlichen Arbeitens. Zudem sind auch hierbei die wert- und interessengeleiteten Entscheide nicht transparent. ${ }^{8}$ Der verbreitete Eindruck einer gewissen Beliebigkeit, ja Orientierungslosigkeit geographischen Arbeitens ist deshalb verständlich - wenn auch gewiß nicht immer gerechtfertigt.

Eine offenere Diskussion über unsere Leitbilder ist also notwendig. Was bedeutet "Fortschritt der Geographie? ${ }^{9}$ Zunächst einmal meint Fortschritt sicher nicht einfach ein quantitatives Anwachsen von Forschungsarbeiten, Personalstellen, Absolventen; vielmehr eine qualitative Entfaltung auf ein Ziel hin gerichtet. Im folgenden sollen dazu einige Überlegungen angestellt werden. Ausgehend von fünf Prinzipien von Wissenschaftlichkeit als Oberziel unserer Arbeit werde ich einige Schlußfolgerungen ziehen, zunächst solche allgemein-methodologi- scher Natur (also zur formalen Perspektive), dann einige disziplin-spezifische Überlegungen, d. h. zur inhaltlichen Perspektive.

\section{Prinzipien der Wissenschaftlichkeit}

Forschung und Lehre müssen sich orientieren (und messen lassen) an bestimmten Qualitätskriterien; sie müssen relevant sein. Ich unterscheide fünf $\mathrm{Re}$ levanz-Kriterien, welche kumulativ erfüllt sein sollten. ${ }^{10}$ Sie sind notwendige Bedingungen wissenschaftlichen Arbeitens.

Erstens geht es um "wissenschaftliche Relevanz» im engeren Sinne. Damit meine ich einerseits in formaler Hinsicht eine Orientierung an den wissenschaftstheoretischen Hauptströmungen analytischer bzw. nicht-analytischer Richtung, das Beachten der üblichen Standards der jeweiligen methodologischen Perspektive (was selbstverständlich bei einem positivistischen Ansatz nicht dasselbe ist wie z. B. bei einem handlungstheoretischen Ansatz). Dabei gilt allgemein, daß deskriptiven oder Basis-Sätzen nur propädeutische Bedeutung zukommt. Zudem soll die Arbeit inhaltlich fruchtbar, innovativ sein: neue Einsichten sollen gewonnen, bestehende konsolidiert werden.

Zweitens geht es um «didaktische Relevanz»: das Primärwissen der Forschungspraxis muß umgesetzt werden, es nützt nichts, wenn es nur in den Forschungsinstituten bleibt. Durch die nach wie vor gültige Verknüpfung von Forschung und Lehre ist damit primär die Hochschulausbildung angesprochen: welches ist der Bezug zwischen Forschungsprojekten und Lernzielen bzw. Lehrangebot? Was für Kompetenzen sollen gefördert werden? Darüber hinaus sind aber weitere Zielgruppen von Bedeutung: die Schule, die Praxis, die Öffentlichkeit im weitesten Sinne. Sie alle sollen neue wissenschaftliche Erkenntnisse sinnvoll und fruchtbar einsetzen können. Umsetzung heißt damit auch Förderung der Kommunikationsfähigkeit gegenüber Partnern außerhalb der Hochschulen. Man muß, um verständlich zu sein, auf die Bedürfnisse und die Sprache dieser Partner eingehen können.

Drittens geht es um "gesellschaftliche Relevanz»: Wissenschaft muß sich auch an den Bedürfnissen und Erwartungen der Gesellschaft orientieren - ein mittlerweile abgestandenes, aber trotzdem nach wie vor brisantes Thema. Eine Diskussion darüber wird auch immer wieder von neuem nötig sein, weil es darum geht, in einem Wust von Spannungsfeldern den rechten Weg zu finden. Lehre und Forschung stehen stets in einer ganz bestimmten Relation zum gesellschaftlichen Umfeld. Eine "anerkannte» Disziplin nimmt in genügendem Maß Bezug auf diese Situation. Einige der damit verbundenen Spannungsfelder will ich kurz in Erinnerung rufen: 
- Ganz handfest ist der Hinweis darauf, daß die Wissenschaft von der Gesellschaft finanziert wird. Oft fällt es schwer, die Grenze zwischen notwendiger «Narrenfreiheit» und leichtfertigem Mißbrauch von Privilegien zu ziehen.

- In einer pluralistischen, fragmentierten Gesellschaft ist die Frage zu stellen: um welche Gesellschaft, welche Gruppen, welche Schichten soll es denn gehen? Und wer definiert ihre Bedürfnisse?

- Von Bedeutung ist auch der Zeithorizont: geht es um kurzfristige Relevanz, um die gerade heute hochaktuellen Fragen oder eher um Probleme, die erst längerfristig voll durchschlagen werden? Wieweit hat die Wissenschaft die Aufgabe eines Frühwarnsystems? Wieweit hat sie überhaupt antizipatorische Kompetenz?

Letztlich geht es wohl stets um das Spannungsfeld zwischen Idealismus einerseits und Opportunismus andererseits. Dabei verdeckt die Vokabel Pragmatismus den jeweils gangbaren Weg mehr, als daß sie ihn aufzeigt. Dennoch: gesellschaftliche Relevanz könnte auch als "pragmatische Relevanz» bezeichnet werden, denn nicht alles, was je von Bedeutung sein könnte, sondern nur was durch die gesellschaftlichen Entscheidungsprozesse verwertbar ist, ist schließlich von Nutzen.

Damit bin ich bereits beim vierten Kriterium, der «ethischen Relevanz». Sie ist ein Korrektiv zur wissenschaftlichen Relevanz wie auch zur gesellschaftlichen bzw. pragmatischen Relevanz. Nicht alles, was sich erforschen läßt, nicht alles, was die Gesellschaft wissen (oder auch verschweigen) möchte, ist «gut». Ethische Relevanz meint also, das eigene Tätigsein als Wissenschafter zu verantworten. Die ethische Dimension in derWissenschaft ist Ausdruck davon, daß diese eingebunden ist ins volle Leben. Ethik läßt sich nicht einfach ausblenden, gerade auch nicht aus der Wissenschaft. Und die Freiheit der Forschung, wird sie dadurch nicht beschnitten? Wohl nicht, wenn es eine verantwortete Freiheit ist, wenn sie nicht als grenzenlos mißverstanden wird.

Wie soll der Wissenschafter diese Verantwortung wahrnehmen? Damit stellt sich die Frage nach der persönlichen Orientierung, die Frage nach der persönlichen Haltung; wir könnten auch sagen: die Sinnfrage überhaupt, für jeden einzelnen, was er vertritt. Allerdings: die Forderung nach einer gewissen Transparenz darf nicht zur Normierung führen. Die Diskussion darüber muß verschiedene Ebenen berücksichtigen, die individualethische wie auch die sozialethische Dimension, ferner die sich daraus ergebenden wissenschaftstheoretischen Konsequenzen. Dies sei hier freilich nur angedeutet.

Schließlich das fünfte Kriterium: «fachpolitische Relevanz». Eine Disziplin sollte eine Identität haben, sie sollte minimale Konturen, einen erkennbaren Kerngehalt aufweisen. Das ist in gewissem
Sinne ein Korrektiv zum methodologischen Pluralismus, der ja quer zu den Inhalten verläuft; die Fach-Identität ergibt sich nicht aus ihren Methoden - sie sind zwar nicht universell, aber auch nicht fachspezifisch-exklusiv. Die Identität kann sich aber auch nicht aus einem exklusiven Realbereich ergeben, aus dem vielzitierten "Gegenstand der Wissenschaft» im Sinne eines Realobjektes - aus dem einfachen Grunde, weil die Erkenntnisbereiche der wissenschaftlichen Disziplinen nicht die realen Gegenstandsbereiche selbst sind, sondern immer Konstrukte, Fragen über Sachverhalte in der «realen» Welt. Die Identität, der Kerngehalt einer Disziplin ergibt sich also aus ihren Fragestellungen, aus ihren Perspektiven über die "Welt»." Doch wie diffus, wie prägnant darf/soll eine Disziplin sein? Eine Antwort darauf ist wohl nur denkbar in. Relation zu andern Disziplinen, zur aktuellen Notwendigkeit einer Identifikation. Diese Notwendigkeit erscheint heute - bei verstärkter Konkurrenz um Forschungsgelder, Curricula und Arbeitsplätze - dringlicher als auch schon.

Zusammenfassend erkennen wir ein erweitertes Wissenschaftsverständnis, eine Ausweitung des Wissenschaftsbegriffes. Wissenschaft ist nicht einfach «wissenschaftlich» im Sinne gültiger wissenschaftstheoretischer Aussagen, sondern sie muß auch verständlich, brauchbar, verantwortbar und prägnant sein. Wissenschaft basiert auf den fünf Prinzipien

- Wissenschaftlickeit (i. e. S.)

- Verständlichkeit

- Brauchbarkeit

- Verantwortung

- Identität

Sie sind das Oberziel jeglichen wissenschaftlichen Arbeitens.

Wir sollten dieses erweiterte Wissenschaftsverständnis akzeptieren, und wir sollten uns an diesen fünf Prinzipien orientieren, wenn wir über ein Leitbild für die Geographie diskutieren.

\section{Schlußfolgerungen I: Formale Perspektive}

Wenn wir versuchen, das bisher Gesagte umzusetzen, wenden wir uns zunächst der Frage zu, wie èine Disziplin ihre Themen behandeln soll. Nennen wir diese allgemein-methodologischen Schlußfolgerungen die «formale Perspektive». Und lassen wir uns (ich wiederhole es) leiten von der Erwartung, unsere Disziplin sollte vermehrt Handlungsbezug aufweisen, vermehrt zu Problemlösungen beitragen; nicht nur wegen des Arbeitsmarktes, sondern auch im Blick auf die beiden Kriterien der "Brauchbarkeit» und der «Verantwortung». Drei Forderungen ergeben sich daraus: 
1. Wir brauchen eine ausreichende Theoriebasis, die Geographie muß sich vermehrt theorieorientiert entwickeln - nicht als Selbstzweck, sondern weil uns nur eine ausreichende Theoriebasis in die Lage versetzt, Transferwissen bereitzustellen; nur allgemein-gültige Sätze können auf spezifische Problemsituationen übertragen und angewendet werden. Zudem ist es nur so möglich, ausreichend gesicherte Aussagen über zukünftige Sachverhalte zu machen, Projektionen zu entwerfen. ${ }^{12}$ Und jeder Handlungsbezug hat ja immer ganz zentral eine zukunftsorientierte Dimension. $\mathrm{Da} \beta$ demgegenüber casuistisches Vorgehen weder rationell noch zeitgerecht ist und zudem (bei öffentlichen Aufgaben) rechtlich problematisch sein kann (Legalitätsprinzip, Gleichheitsgebot), sei hier nur am Rande vermerkt.

2. Das Normendefizit muß behoben werden; «wertfreie» Wissenschaft ist eine untaugliche Fiktion. Aus Fakten allein lassen sich noch keine handlungsbezogenen Schlüsse ziehen; es gibt keine "angewandte Wissenschaft» ohne normative Bezüge. Vielmehr sind aus Ziel-Mittel-Hierarchien wirksame Maßnahmen abzuleiten. Dabei kommt normativen Modellen (Entscheidungsmodellen) eine große Bedeutung $\mathrm{zu}$; sie sollen reale Entscheidungsmechanismen gültig abbilden und die Schnittstellen zwischen Fakten und Werten deutlich machen. Selbstverständlich darf die hier zentrale Frage nach der Normenbasis, nach der Legitimation, nicht vernachlässigt werden.

3. Schließlich dürfen wir die operative Ebene nicht vergessen. Es geht ja darum, Entscheidungsgrundlagen umzusetzen in Handeln, in konkrete Maßnahmen. Hier ist also der direkte Praxisbezug angesprochen, und es wäre zu prüfen, ob dieser Praxisbezug nicht verstärkt Teil der Ausbildung sein müßte (im Sinne handlungsbezogener Lehrveranstaltungen), ob er nicht sozusagen als "didaktische Matrix» gelten müßte. Ich plädiere dafür, daß wirTheorie und Praxis (und bei gesellschaftlichem Handeln bedeutet dies: Politik) nicht voneinander abkoppeln dürfen. In andern Disziplinen ist das selbstverständlich, da stehen sie eng verbunden nebeneinander, oft als Teile derselben Lehrveranstaltung. Das sollte uns wenn wir wirklich am Praxisbezug interessiert sind - als Leitbild dienen.

Ob dieses neue Bild einer Wissenschaft, welche auf Theorien (bzw. Theorieansätze) hinzielt und darauf basierend normativ gestützte Entscheidungsgrundlagen bereitstellt und sich (sogar aktiv) an der Gestaltung beteiligt - ob dieses Bild «richtig» ist, das ist eine Frage der persönlichen Überzeugung jedes Wissenschafters, jedes Hochschullehrers. Aber eins ist sicher: die Bedeutung, die heute in der Gesellschaft einer Disziplin beigemessen wird, diese Be- deutung ist desto größer, je prägnanter ihr Beitrag ausfällt auf explikativer, normativer und operativer Ebene. Diese drei Ebenen lassen sich weder voneinander noch von ihrer deskriptiven Basis isolieren. Wer bewerten und entscheiden will ohne Theoriebasis, der irrt sosehr wie derjenige, der Deskription allein für eine wissenschaftliche Spitzenleistung hält.

\section{Schlußfolgerungen II: Inhaltliche Perspektiven}

Nun ist noch die Frage zu beantworten, was fürThemen die Geographie bearbeiten soll. $\mathrm{Zu}$ dieser inhaltlichen Perspektive kann ich mich an dieser Stelle knapp fassen, nachdem schon heute vormittag im Referat von Frau Hantschel dazu sehr vieles gesagt wurde, was ich (durchaus zustimmend) nicht wiederholen möchte. Selbstverständlich müssen diese Überlegungen besonders die Forderung nach fachpolitischer Relevanz, nach «Identität» also, berücksichtigen, freilich ohne die übrigen Kriterien zu vernachlässigen. Nun, worin könnte denn dieser identifizierende Kernbereich der Geographie bestehen, bei aller Diffusität sozusagen?

Meines Erachtens besteht der Kernbereich der Geographie in einer komplex-räumlichen, prozeß-räumlichen Perspektive, in einer räumlich-integrativen Perspektive oder schlicht: im Raumbezug. Damit meine ich die zentrale Bedeutung der Vernetzung von Mensch und Umwelt, von Wirtschaft, Gesellschaft und Natur mit ihrer Raumwirksamkeit, also mit einem Konkretisierungsgrad, der andern ähnlichen Ansätzen (z. B. den 1-Punkt-Modellen in der Umweltökonomie) mangelt. Umgekehrt besteht auch eine klare Abgrenzung gegenüber räumlich differenzierten, aber sachdimensional nur einschichtigen Ansätzen (z. B. in der klassischen Regionalökonomie). Auf der normativ-operativen Ebene geht es um die Gestaltung unseres Lebensraumes - sicher nicht eine "mythische Klammer» (P. SEDLACEK), sondern von den Betroffenen sehr konkret erlebbar. Raum als Prozeßfeld hat also eine dreifache Bedeutung: er ist Metapher für die konkrete Interaktion verschiedener Kräfte, und er ist materielles Substrat wie auch Ergebnis solcher Prozesse.

$\mathrm{Da} \beta$ diese komplexen, ressort-übergreifenden $\mathrm{Zu}$ sammenhänge in der «realen» Welt heute von eminenter Bedeutung sind, ist wohl unbestreitbar. Und so erscheint.es als sinnvoll, wenn nicht gar notwendig, solchen Zusammenhängen mit wissenschaftlichem Anspruch nachzuspüren. Dazu gehört sicher auch eine stets wiederkehrende Anstrengung, das lineare Denken zu überwinden und die Segmentierung der "vernetzten Welt», in der wir leben, und die damit auftauchenden Probleme zu überwinden - eine Segmentierung, die wir ja dem analytischen Ansatz verdanken. Im Sinne der Komplementari- 
täts-Idee sind aus der Berührung von analytischen und makroskopischen Perspektiven neue Einsichten zu erwarten.

Das «Dualismus-Dogma», in der Geographie seit bals 20 Jahren sehr explizit vorgetragen, hat sich als wissenschaftstheoretische Sackgasse erwiesen. Wir haben teuer bezahlt dafür, indem wir den Anschluß an das «Vernetzte Denken» fast verpaßt haben (von einer Initiative ganz zu schweigen!). Ist es denkbar, daß «Ökonomie» und "Ökologie» je für sich mit höchster Akribie (und höchst abstrakt) seziert werden, ihr Zusammenwirken (besser: Zusammenprallen) aber von der Wissenschaft bewußt ignoriert wird? Dazu muß man schließlich auch einmal fragen, wer denn immer noch ein Interesse daran hat, Wirtschaft und Gesellschaft von der Natur abzukoppeln?

\section{Offene Fragen}

Das hier nur sehr grob skizzierte Leitbild einer normativ orientierten, integrativen Geographie wirft eine Reihe von Fragen auf, von denen ich einige wenige kurz streifen möchte. Eine eingehende Diskussion darüber erscheint vordringlich.

Der intregrative Ansatz ist nicht lediglich «alter Wein in neuen Schläuchen», nämlich das Landschaftskonzept in aktueller Verpackung. Wer so argumentiert, übersieht einen grundlegenden Unterschied zwischen beiden Ansätzen: es geht keineswegs darum, eine "Gesamtsynthese» zu machen, ein deskriptives «Gesamtmodell» diffuser Entitäten aufzubauen; das wäre (neben andern Fragwürdigkeiten) ein überrissener Anspruch, der unerfüllbar bleiben muß. Vielmehr geht es um die Lösung ganz konkreter Probleme im Kontaktbereich von Teilen unserer Welt, die durch die Entwicklung derWissenschaften in verschiedene Schubladen geraten sind. Dazu müssen wir relevante Kräfte und Faktoren dieses Zusammenwirkens von Ökonomie und Ökologie herausarbeiten, und zwar insbesondere solche Faktoren, welche im Raum sichtbar werden. Dabei müssen wir uns jeweils auf einige wesentliche Elemente konzentrieren - nur schon deshalb, weil auch Problemlösungen kein «allumfassendes Gesamt» sind, sondern ganz konkrete Maßnahmen. Trotzdem: ein Spannungsfeld zwischen ganzheitlichem (holistischem) Verständnis unserer Lebenswelt und der Notwendigkeit selektiven Vorgehens wird stets bestehen bleiben.

Damit ist auch ein wissenschaftstheoretisches, methodisches und wissenschaftspolitisches Problemfeld angesprochen: in einer analytisch orientierten, ja dominierten Welt werden sich makroskopoische
Ansätze wohl noch während einiger Zeit schwer tun. Vorderhand nur in Umrissen skizziert und methodisch wenig entwickelt, ohne bereits überzeugende Praxis, mögen sie dem an analytische Spitzenleistungen gewöhnten Zeitgeist nicht genügen. Mit andern Worten: dieses Defizit abzubauen, hier überzeugende und brauchbare Ergebnisse zu entwickeln, ist eine echte (vielleicht sogar: die aktuellste) Forschungsfront, vor allem auch in methodischer Hinsicht. Die Verknüpfungsleistung von Ökonomie und Ökologie ist nicht nur eine didaktische Aufgabe; integrativ orientierte Geographie müßte keine «second-hand»-Disziplin sein. Wegweisende Denkmodelle wie etwa die die Theorie dissipativer Strukturen (J. PRIGOGINE) oder die Synergetik makroskopischer Prozesse (H. HAKEN) sollten rasch adaptiert werden und zur Weiterentwicklung von Fachtheorien führen. Dabei sind Komplementaritäts-Effekte zwischen analytischen Partialtheorien und makroskopischen Modellen zu erwarten.

Diese Vorstellungen stellen ein sehr ambitiöses Programm dar, ein Leitbild für die Geographie als Disziplin, für unsere Identität gegenüber der Öffentlichkeit. Die Hochschulinstitute füllen es aus mit ihrer Arbeit in Forschung und Lehre. Für den Einzelnen bedeutet es, seinen individuellen Platz in diesem großen Rahmen zu finden, ohne die integrative Basisperspektive, den Raumbezug, aus den Augen zu verlieren. Zusammen erst ergibt sich der Leistungsausweis einer ganzen Disziplin.

\section{Zusammenfassung}

Überlegungen zu einem Leitbild für eine zukunftsorientierte Geographie, die in der Lage ist, vermehrt zu Problemlösungen und zur Gestaltung unseres Lebensraumes beizutragen, gehen aus von einem erweiterten Wissenschaftsverständnis. Es basiert auf fünf Prinzipien: Wissenschaftlichkeit im engeren Sinne, Verständlichkeit, Brauchbarkeit, Verantwortung und Identität. Die Vorstellung von einer Einheitsmethodologie im Sinne des Kritischen Rationalismus (bzw. Szientismus) ist abgelöst worden durch einen wissenschaftstheoretischen Pluralismus, insbesondere durch das Nebeneinander von analytischen und makroskopischen Ansätzen. Aktuelle Probleme im Kontaktbereich von Ökonomie und Ökologie stehen zur Lösung an; sie bedürfen einer wissenschaftlichen Fundierung. Problemlösungen im Sinne von Entscheiden über raumwirksame Maßnahmen sind nur möglich auf der Basis ausreichender allgemeiner Sätze (Theorien) und unter Berücksichtigung der maßgebenden Ziel-MittelHierarchien. 
Daraus werden folgende Ziele für den «Fortschritt der Geographie» abgeleitet:

1. Wir müssen eine vertiefte wissenschaftstheoretische Diskussion und einen «Kooperativen Pluralismus» fördern.

2. Wir sollten das erweiterte Wissenschaftsverständnis akzeptieren und uns daran orientieren.

3. Geographie muß sich verstärkt zu einer theorieorientierten Disziplin entwickeln und ihr dreifaches Normendefizit überwinden.

4. Geographie muß sich zu einer engagierten Disziplin im Sinne einer konstruktiv-kritischen Haltung entwickeln.

5. Inhaltlich müssen wir uns orientieren an einem prägnanten Kernbereich, nämlich einer komplexräumlichen, integrativen Perspektive.

Ich bin nicht der Meinung, daß die «diffuse Thematik» unser Hauptproblem ist; vielmehr sind wir oft zu wenig leistungsfähig im Sinne der genannten Zielsetzungen. Die Defizite können nicht durch «Imagepflege» behoben werden, sondern wir müssen durch unsere Arbeit entscheiden, ob Geographie eine «leichte» oder eine «ernstzunehmende» Disziplin sein soll.

\section{Anmerkungen}

1 Damit sind hier natürlich Probleme der Praxis, der Alltagswelt und der Politik gemeint, und nicht rein kognitive Probleme.

2 Vgl. dazu auch A. BUTTIMER (1981/96): «We need more epistemologically rigorous foundations für research and a more open discussion of values before we can identify a credible core for the discipline of geography today."

3 Vgl. dazu J. SPECK (1980/726ff.; 771 ff.)

${ }^{4} \mathrm{Vgl}$. dazu den folgenden Abschnitt.

5 Vgl. dazu R. J. JOHNSTON 1983.

6 Ein Musterbeispiel dafür ist etwa die Umweltverträglichkeitsprüfung (UVP), bei der prognostische und normative Elemente (System-Technik, Szenario-Technik; BewertungsFunktionen) ganz offensichtlich eine große Rolle spielen.

$7 \mathrm{Vgl}$. dazu besonders D. HÖLLHUBER (1981)

8 Vgl. dazu D. BARTELS (1970)

${ }^{9}$ Vgl. dazu M. J. WISE (1977)
10 Vgl. dazu neben D. BARTELS (1970) auch B. WERLEN (1986)

11 So wie etwa die Ökonomie letztlich immer von der Knappheit und ihrer angemessenen Beachtung durch "optimales Tun" handelt.

12 Das es sich hierbei nicht um Prognosen im naturwissenschaftlichen Sinne handeln kann, braucht wohl nicht speziell hervorgehoben zu werden.

\section{Literatur}

BARTELS, D. (1970): Zwischen Theorie und Metatheorie. In: Geographische Rundschau 22/451-457/1970.

BOESCH, M. (1981): Geographie zwischen Choristik und Orthochorie. Ansätze einer methodologischen Standortbestimmung. In: SNG-Jahrbuch 1979. Basel 1981.

BOESCH, M. (1986): On the lacking Normative power of Geography. In: IGU Working Group "Landscape Synthesis», Abstracts, Symposium Dessau 1985. Halle 1986.

BUTTIMER, A. (1981): On people, paradigms, and "progress". In: STODDART D. R. (ed) Geography, Ideology, and Social Concern. Oxford 1981.

CAPRA, F. (1982): Wendezeit - Bausteine für ein neues Weltbild. (The Turning Point). Bern 811984.

COFFEY, W. J. (1981): Geography - Towards a General Spatial Systems Approach. London 1981.

DAUM, E./SCHMIDT-WULFFEN, W.-D. (1980): Erdkunde ohne Zukunft? Konkrete Alternativen zu einer Didaktik der Belanglosigkeiten, Paderborn 1980.

FERGUSON, M. (1980): Die Sanfte Verschwörung. Persönliche und Gesellschaftliche Transformation im Zeitalter des Wassermanns. (The Aquarian Conspiracy). Basel 311983.

HANTSCHEL, R. (1984): Neuere Ansätze in der Anthropogeographie. In: Geographica Helvetica 39 (3) / 137-143/1984.

HÖLLHUBER, D. (1981): Probleme der künftigen Entwicklung der Kernstädte in der Bundesrepublik Deutschland und ihre Behandlung in geographischen Untersuchungen. In: Geographische Zeitschrift 69 (4)/241-266/1981.

JOHNSTON, R. J. (1983): Philosophy and Human Geography. An Introduction to Contemporary Approaches. Ĺondon 1983.

POSTMAN, N. (1982): Das Verschwinden der Kindheit. Frankfurt a. M. 911985.

WERLEN, B. (1986): Zwischen Metatheorie, Fachtheorie und Alltagswelt. Im Druck.

WISE, M. J. (1977): On progress and geography. In: Progress in Human Geography 1 (1)/1-10/1977.

SPECK, J. (1980): Handbuch wissenschaftstheoretischer Begriffe. 3 Bände (= UTB 966/967/968). Göttingen 1980ff. 